Pacific Journal of Mathematics

THE FEFFERMAN-STEIN DECOMPOSITION OF SMOOTH
FUNCTIONS AND ITS APPLICATION TO $H^{p}\left(\mathrm{R}^{n}\right)$ 


\title{
THE FEFFERMAN-STEIN DECOMPOSITION OF SMOOTH FUNCTIONS AND ITS APPLICATION TO $H^{p}\left(\mathbf{R}^{n}\right)$
}

\author{
AKIHITo UCHIYAMA
}

We show the "Fefferman-Stein decomposition" of smooth bump functions. As an application of this we get one result about the singular integral characterization of $H^{p}\left(\mathbf{R}^{n}\right)$. Our method does not use subharmonicity.

\section{TABLE OF CONTENTS}

1. Introduction

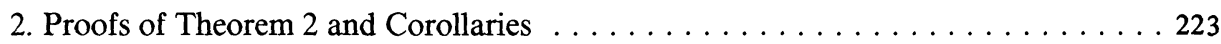

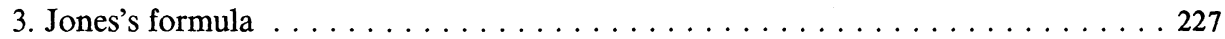

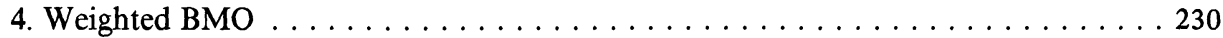

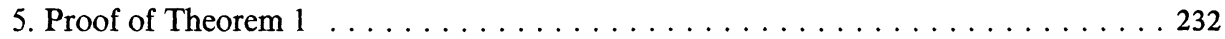

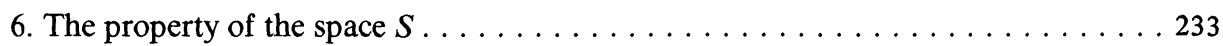

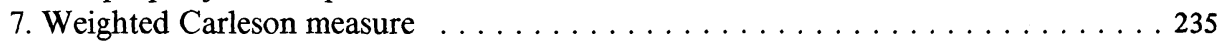

8. The decomposition of weighted BMO functions $\ldots \ldots \ldots \ldots \ldots \ldots \ldots \ldots \ldots$

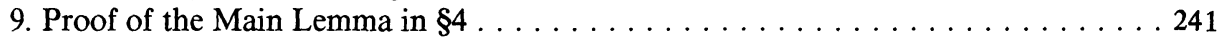

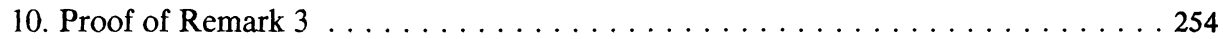

1. Introduction. In this paper functions considered are complexvalued unless otherwise explicitly stated. Cubes considered have sides parallel to the coordinate axes. For a function $f(x) \in L_{\mathrm{loc}}^{1}\left(\mathbf{R}^{n}\right)$, let

$$
\|f\|_{\mathrm{BMO}}=\sup _{I} \int_{I}\left|f(x)-f_{I}\right| d x /|I|,
$$

where the supremum is taken over all cubes in $\mathbf{R}^{n},|I|$ denotes the Lebesgue measure of $I$ and

$$
f_{I}=\int_{I} f(x) d x /|I|
$$

A function $f(x)$ is said to belong to $\mathrm{BMO}\left(\mathbf{R}^{n}\right)$ if $\|f\|_{\mathrm{BMO}}<+\infty$.

Let $\theta_{1}(\xi), \ldots, \theta_{m}(\xi) \in C^{\infty}\left(S_{n-1}\right)$, where

$$
S_{n-1}=\left\{\xi \in \mathbf{R}^{n}:|\xi|=1\right\}
$$

and

$$
|\xi|=\left|\left(\xi_{1}, \ldots, \xi_{n}\right)\right|=\left(\sum_{j=1}^{n} \xi_{j}^{2}\right)^{1 / 2}
$$


For $h \in L^{2}\left(\mathbf{R}^{n}\right)$ let

$$
K_{j} h=\left(\theta_{j}(\xi /|\xi|) \hat{h}(\xi)\right)^{\vee}, \quad j=1, \ldots, m,
$$

where ${ }^{\wedge}$ and ${ }^{`}$ are the Fourier and inverse Fourier transforms. As is well known [see Stein [29] p. 75], there exist $\alpha_{J} \in \mathbf{C}$ and $\Omega_{j}(x) \in C^{\infty}\left(S_{n-1}\right)$ such that

$$
\int_{|x|=1} \Omega_{j}(x)=0
$$

and

$$
K_{j} h(x)=\alpha_{j} h(x)+\text { P.V. } \int \Omega_{j}\left(\frac{x-y}{|x-y|}\right)|x-y|^{-n} h(y) d y
$$

for any $h \in L^{2}\left(\mathbf{R}^{n}\right)$. For $g \in L^{\infty}\left(\mathbf{R}^{n}\right)$ let

$\tilde{K}_{j} g(x)=\alpha_{j} g(x)$

$$
+ \text { P.V. } \int\left\{\Omega_{j}\left(\frac{x-y}{|x-y|}\right)|x-y|^{-n}-\Omega_{J}\left(\frac{-y}{|y|}\right)|y|^{-n} \chi_{\{|b|>1\}}\right\} g(y) d y
$$

where $\chi_{E}$ denotes the characteristic function of a set $E \subset \mathbf{R}^{n}$. In [32], the author showed

THEOREM A. If

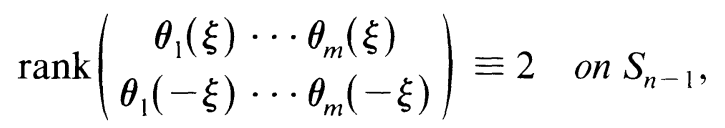

then for any $f \in \operatorname{BMO}\left(\mathbf{R}^{n}\right)$ there exist $g_{1}, \ldots, g_{m} \in L^{\infty}\left(\mathbf{R}^{n}\right)$ such that

$$
f=\sum_{j=1}^{m} \tilde{K}_{j} g_{j} \quad \text { (modulo constants) }
$$

and

$$
\sum_{j=1}^{m}\left\|g_{j}\right\|_{\infty} \leq C_{1.1}\|f\|_{\mathrm{BMO}}
$$

where $C_{11}$ is a constant depending only on $\theta_{1}, \ldots, \theta_{m}$.

REMARK 1. The case when $K_{1}, \ldots, K_{n+1}$ are the Riesz transforms and the identity operator is the case considered by C. Fefferman [13] and C. Fefferman-Stein [14]. 
REMARK 2. In [32] we assumed that $f$ has compact support. But this restriction can be removed.

Consequently, if (1.1) is satisfied, then the singular integral operators $K_{1}, \ldots, K_{m}$ characterize $H^{1}\left(\mathbf{R}^{n}\right)$. In this paper, we continue this research.

In the following, $\mathbf{f}(x)=\left(f_{1}(x), \ldots, f_{m}(x)\right)$ and $\mathbf{g}(x)$ denote $\mathbf{C}^{m}$-valued functions. We use the following notations:

$$
\begin{aligned}
|\mathbf{f}(x)| & =\left(\sum_{j=1}^{m}\left|f_{j}(x)\right|^{2}\right)^{1 / 2}, \\
\mathbf{K} h(x) & =\left(K_{1} h(x), \ldots, K_{m} h(x)\right), \\
\mathbf{K} \cdot \mathbf{f}(x) & =\sum_{j=1}^{m} K_{j} f_{j}(x), \\
\mathbf{K}^{*} \cdot \mathbf{f}(x) & =\sum_{j=1}^{m} K_{j}^{*} f_{j}(x),
\end{aligned}
$$

where $K_{j}^{*} h(x)=\left(\bar{\theta}_{j}(\xi /|\xi|) \hat{h}(\xi)\right)(x) . I(x, t)$ denotes a cube in $\mathbf{R}^{n}$ with center $x$ and side length $t$.

DeFINITION 1.1. Let

$$
S=\left\{\mathbf{f} \in L^{2}\left(\mathbf{R}^{n}, \mathbf{C}^{m}\right): \mathbf{K}^{*} \cdot \mathbf{f}(x) \equiv 0\right\},
$$

where $L^{2}\left(\mathbf{R}^{n}, \mathbf{C}^{m}\right)$ denotes the set of $\mathbf{C}^{m}$-valued functions $\mathbf{f}(x)$ with $f_{1}, \ldots, f_{m} \in L^{2}\left(\mathbf{R}^{n}\right)$.

Definition 1.A. [Coifman-Rochberg [9].] For a real-valued function $f \in L_{\mathrm{loc}}^{1}\left(\mathbf{R}^{n}\right)$, let

$$
\|f\|_{\mathrm{BLO}}=\sup _{I} \int_{I} f(x)-\inf _{y \in I} f(y) d x /|I|,
$$

where $I$ is taken over all cubes in $\mathbf{R}^{n}$. A function $f(x)$ is said to belong to $\operatorname{BLO}\left(\mathbf{R}^{n}\right)$ if $\|f\|_{\text {BLO }}<+\infty$. [Note that $\|\cdot\|_{\mathrm{BLO}}$ is not a norm.]

Our main result is the following.

THeOReM 1. Suppose that (1.1) holds. Let $\mathbf{f} \in C^{1}\left(\mathbf{R}^{n}, \mathbf{C}^{m}\right)$,

$$
|\mathbf{f}(x)| \leq(1+|x|)^{-n-1},
$$


and

$$
\left|\frac{\partial}{\partial x_{J}} \mathbf{f}(x)\right| \leq(1+|x|)^{-n-2}, \quad j=1,2, \ldots, n .
$$

Let $w(x)$ be a nonnegative function defined on $\mathbf{R}^{n}$ such that

$$
\|-\log w\|_{\mathrm{BLO}} \leq c_{0} .
$$

Then there exists $\mathbf{g} \in L^{2}\left(\mathbf{R}^{n}, \mathbf{C}^{m}\right)$ such that

$$
\mathbf{f}-\mathbf{g} \in S
$$

and that

$$
|\mathbf{g}(x)| \leq C_{1.2} w(x)\left(\int_{I(0,1)} w(y) d y\right)^{-1}(1+|x|)^{-n-1 / 2},
$$

where $c_{0}$ and $C_{1.2}$ are positive constants depending only on $\theta_{1}, \ldots, \theta_{m}$.

REMARK 3. If $\mathbf{f}(x)$ is $\mathbf{R}^{m}$-valued and if $\theta_{j}(\xi) \equiv \bar{\theta}_{j}(-\xi)$ for $j=1, \ldots, m$, then we can take $\mathbf{g}(x)$ to be $\mathbf{R}^{m}$-valued.

REMARK 4. If we apply Theorem 1 to the case when $K_{1}=$ the identity operator and $\mathbf{f}(x)=(f(x), 0, \ldots, 0)$, then (1.5) implies

$$
f(x)=g_{1}(x)+\sum_{j=2}^{m} K_{j}^{*} g_{j}(x) .
$$

This is the reason why we call Theorem 1 the Fefferman-Stein decomposition of smooth bump functions. The point is the fact that we can dominate $g_{1}, \ldots, g_{m}$ pointwise by a "function" on the right-hand side of (1.6).

The idea of this theorem comes from P. W. Jones's recent work " $L$ " estimate for the $\bar{\partial}$ problem in a half-plane" [25]. We explain the relation between Theorem 1 and Jones's result in $\S 3$.

The proof of Theorem 1 is given in $\S 5$. The Main Lemma in $\$ 4$ is crucial and is itself a partial result related to the Fefferman-Stein decomposition of certain weighted BMO spaces in terms of singular integral operators $K_{1}, \ldots, K_{m}$. The Main Lemma is proved in $\$ \S 6-9$. Its proof is a refinement of the argument in [32].

As a corollary to Theorem 1 , we get one result about the singular integral characterization of $H^{p}\left(\mathbf{R}^{n}\right)$. Let $\psi \in \mathscr{Q}\left(\mathbf{R}^{n}\right)$ be a fixed real-valued function satisfying $\int \psi(x) d x=1$. For $h \in \mathcal{S}^{\prime}\left(\mathbf{R}^{n}\right)$, let

$$
h^{+}(x)=\sup _{t>0}\left|\left(h * \psi_{t}\right)(x)\right|,
$$


where $\psi_{t}(x)=t^{-n} \psi(x / t)$. For $+\infty>p>0$, let

$$
\|h\|_{H^{p}}=\left\|h^{+}\right\|_{L^{p}} .
$$

For $\mathbf{h}=\left(h_{1}, \ldots, h_{m}\right) \in \mathcal{S}^{\prime}\left(\mathbf{R}^{n}\right) \oplus \cdots \oplus \mathcal{S}^{\prime}\left(\mathbf{R}^{n}\right)$, let

$$
\mathbf{h}^{+}(x)=\sup _{t>0}\left|\left(\mathbf{h} * \psi_{t}\right)(x)\right|=\sup _{t>0}\left|\left(\left(h_{1} * \psi_{t}\right)(x), \ldots,\left(h_{m} * \psi_{t}\right)(x)\right)\right| .
$$

It is known that $\|\cdot\|_{H^{p}}$ is essentially independent of the choice of $\psi$. [See C. Fefferman-Stein [14].]

Definition 1.B. For $q>0$ and for a measurable function $f(x)$ let

$$
M_{q} f(x)=\sup _{I \ni x}\left(\int_{I}|f(y)|^{q} d y /|I|\right)^{1 / q}
$$

where $I$ is taken over all cubes containing $x$.

THeOREM 2. If (1.1) holds, then there exist $p_{0} \in(0,1)$ and $C_{13} \in \mathbf{R}$, depending only on $\theta_{1}, \ldots, \theta_{m}$, such that

$$
(\mathbf{K} h)^{+}(x) \leq C_{1.3} M_{p_{0}}\left(M_{1 / 2}(|\mathbf{K} h|)\right)(x)
$$

for any $x \in \mathbf{R}^{n}$ and any $h \in L^{2}\left(\mathbf{R}^{n}\right)$.

REMARK 5. For $h \in L^{2}\left(\mathbf{R}^{n}\right)$ and $\mathbf{h} \in L^{2}\left(\mathbf{R}^{n}, \mathbf{C}^{m}\right)$, let

$$
\begin{aligned}
& h^{++}(x)=\sup _{\substack{t>0, z \in R^{n}:|x-z|<t}}\left|\left(h * P_{t}\right)(z)\right|, \\
& \mathbf{h}^{++}(x)=\sup _{\substack{t>0, z \in R^{n}:|x-z|<t}}\left|\left(\mathbf{h} * P_{t}\right)(z)\right|,
\end{aligned}
$$

where $P_{t}(x)$ is the Poisson kernel, that is,

$$
P_{t}(x)=c_{n} t /\left(|x|^{2}+t^{2}\right)^{(n+1) / 2}, \quad c_{n}=\Gamma((n+1) / 2) / \pi^{(n+1) / 2} .
$$

Then in the above inequality, we can replace $(\mathbf{K} h)^{+}(x)$ by $(\mathbf{K} h)^{++}(x)$.

COROLlaRY 1. If (1.1) holds and if $\max \left(1 / 2, p_{0}\right)<p \leq 1$, then

$$
c_{1.4}\|h\|_{H^{p}} \leq \sum_{j=1}^{m}\|K, h\|_{L^{p}} \leq c_{1.5}\|h\|_{H^{p}}
$$


for any $h \in L^{2}\left(\mathbf{R}^{n}\right)$ and

$$
\begin{aligned}
c_{1.4}\|h\|_{H^{p}} & \leq \sum_{j=1}^{m}\left(\int_{R^{n}}\left|\lim _{t \rightarrow+0} K_{j}\left(h * P_{t}\right)(x)\right|^{p} d x\right)^{1 / p} \\
& \leq c_{1.5}\|h\|_{H^{p}}
\end{aligned}
$$

for any $h \in H^{p}\left(\mathbf{R}^{n}\right)$, where $c_{1.4}$ and $c_{1.5}$ are positive constants depending only on $\theta_{1}, \ldots, \theta_{m}$ and $p$.

RemarK 6. For $h \in H^{p}\left(\mathbf{R}^{n}\right), p<1$, we define $h * P_{t}$ by $\left(\hat{h}(\xi) \hat{P}_{t}(\xi)\right)^{\check{2}}$, which is known to belong to $L^{1}\left(\mathbf{R}^{n}\right) \cap L^{\infty}\left(\mathbf{R}^{n}\right) \cap C\left(\mathbf{R}^{n}\right)$. It is also known that for any $h \in H^{p}\left(\mathbf{R}^{n}\right), \lim _{t \rightarrow+0} K_{j}\left(h * P_{t}\right)(x)$ exists almost everywhere. [See Stein [29] p. 201.]

REMARK 7. Inequality (1.7) with $p=1$ holds for any $h \in \mathcal{S}^{\prime}\left(\mathbf{R}^{n}\right)$, whose Fourier transform is an integrable function on some neighborhood of the origin, if we define $K_{j} h=\left(\theta_{j} \hat{h}\right)^{2}$ in the sense of distributions and if we define

$$
\left\|K_{j} h\right\|_{L^{1}}=+\infty
$$

for the distribution $K_{j} h$ that does not belong to $L^{1}\left(\mathbf{R}^{n}\right)$. [In Corollary 1 of [32], we showed the above. But the statement in [32] was somewhat ambiguous.]

As another application of Theorem 2, we get the following extension of the results of Csereteli, Gundy and Varopoulos. [See [12], [18] and [34].]

COROllary 2. Let

$$
\sum_{j=1}^{m}\left|\theta_{j}(\xi)-\theta_{j}(-\xi)\right| \neq 0 \quad \text { for any } \xi \in S_{n-1} .
$$

Let $h$ be a finite complex measure on $\mathbf{R}^{n}$ and let $d h=f d x+d s$, where $f \in L^{1}\left(\mathbf{R}^{n}\right)$ and $s$ is singular. Then

$$
\liminf _{\lambda \rightarrow+\infty} \lambda\left|\left\{x \in \mathbf{R}^{n}: \sum_{j=1}^{m}\left|\lim _{t \rightarrow+0} K_{j}\left(h * P_{t}\right)(x)\right|>\lambda\right\}\right| \geq C_{1.6}\|s\|_{M},
$$

where $C_{1.6}$ is a positive constant depending only on $\theta_{1}, \ldots, \theta_{m}$ and where $\|s\|_{M}$ is the total variation of $s$ on $\mathbf{R}^{n}$. 
REMARK 8. It is known that for any finite measure $h$

$$
\lim _{t \rightarrow+0} K_{j}\left(h * P_{t}\right)(x)
$$

exists almost everywhere.

Proofs of Theorem 2 and corollaries are given in $\$ 2$.

Notation. A dyadic cube is a cube of the form

$$
\prod_{j=1}^{n}\left[k_{j} 2^{-k},\left(k_{j}+1\right) 2^{-k}\right],
$$

where $k_{1}, \ldots, k_{n}$ and $k$ are integers. For a cube $I, x_{I}, l(I)$ and $Q(I)$ denote the center of $I$, the side length of $I$ and

$$
\left\{(x, t) \in \mathbf{R}^{n+1}: x \in I, t \in(0, l(I))\right\},
$$

respectively. For $\alpha>0, \alpha I$ denotes a cube concentric with $I$ and with $l(\alpha I)=\alpha l(I) . \sum_{2 m-1}$ denotes $\left\{\boldsymbol{\nu}=\left(\nu_{1}, \ldots, \nu_{m}\right) \in \mathbf{C}^{m}: \sum_{j=1}^{m}\left|\nu_{j}\right|^{2}=1\right\} .|\boldsymbol{\nu}|$ denotes $\left(\sum_{j=1}^{m}\left|\boldsymbol{\nu}_{j}\right|^{2}\right)^{1 / 2}$. For $\boldsymbol{\nu} \in \mathbf{C}^{m} \backslash\{\boldsymbol{0}\}, U(\boldsymbol{\nu})$ denotes $\boldsymbol{\nu} /|\boldsymbol{\nu}|$. [For the sake of convenience, let $U(\mathbf{0})=(1,0, \ldots, 0)$.] For $\boldsymbol{\nu}$ and $\boldsymbol{\mu} \in \mathbf{C}^{m},\langle\boldsymbol{\nu}, \boldsymbol{\mu}\rangle$ denotes $\sum_{j=1}^{m}\left(\operatorname{Re} \nu_{j} \operatorname{Re} \mu_{j}+\operatorname{Im} \nu_{j} \operatorname{Im} \mu_{j}\right)$, i.e., the inner product in $\mathbf{R}^{2 m}$. For $\theta \in C^{\infty}\left(S_{n-1}\right)$ and $\xi \in \mathbf{R}^{n} \backslash\{0\}, \theta(\xi)$ denotes,$\theta(\xi /|\xi|)$. The letter $C$ denotes various positive constants depending only on $\theta_{1}, \ldots, \theta_{m}$.

Acknowledgements. The author would like to express his deep gratitude to Professor P. W. Jones and the Department of Mathematics at the University of Chicago for their kindness during the academic year 19811982.

\section{Proofs of Theorem 2 and Corollaries.}

LEMMA 2.A. [See Coifman-Rochberg [9].] If $h(x) \neq 0$ and if $M_{1} h(x) \neq$ $+\infty$, then

$$
\left\|\log M_{1} h\right\|_{\mathrm{BLO}} \leq C_{2.1} \text {. }
$$

Proof of Theorem 2. By dilation and translation the proof of Theorem 2 can be reduced to the inequality

$$
\left|\int \mathbf{K} h(x) \psi(x) d x\right| \leq C_{1.3} M_{p_{0}}\left(M_{1 / 2}(|\mathbf{K} h|)\right)(0) \text {. }
$$


Put $\varepsilon=c_{0} / 2 C_{2.1}$. Take any $\boldsymbol{\nu} \in \Sigma_{2 m-1}$. Applying Theorem 1 to $\mathbf{f}(x)$ $=\psi(x) \boldsymbol{\nu}$ and $w(x)=M_{1 / 2}(|\mathbf{K} h|)(x)^{-\varepsilon}$, we get $\mathbf{g}(x)$ such that

$$
\mathbf{K}^{*} \cdot(\psi \boldsymbol{\nu}-\mathbf{g}) \equiv 0
$$

and such that

$$
\begin{aligned}
|\mathbf{g}(x)| \leq & C M_{1 / 2}(|\mathbf{K} h|)(x)^{-\varepsilon}\left(\int_{I(0,1)} M_{1 / 2}(|\mathbf{K} h|)(y)^{-\varepsilon} d y\right)^{-1} \\
& \times(1+|x|)^{-n-1 / 2} \\
\leq & C M_{1 / 2}(|\mathbf{K} h|)(x)^{-\varepsilon} M_{1 / 2}(|\mathbf{K} h|)(0)^{\varepsilon}(1+|x|)^{-n-1 / 2} .
\end{aligned}
$$

Thus

$$
\begin{aligned}
& \left|\int \mathbf{K} h(x) \psi(x) d x \cdot \boldsymbol{\nu}\right|=\left|\int \mathbf{K} h(x) \cdot \mathbf{g}(x) d x\right| \leq \int|\mathbf{K} h(x)||\mathbf{g}(x)| d x \\
& \leq C M_{1 / 2}(|\mathbf{K} h|)(0)^{\varepsilon} \int M_{1 / 2}(|\mathbf{K} h|)(x)^{1-\varepsilon}(1+|x|)^{-n-1 / 2} d x \\
& \leq C M_{1-\varepsilon}\left(M_{1 / 2}(|\mathbf{K} h|)\right)(0) .
\end{aligned}
$$

[In the first and the second formulae of the last string of inequalities, · denotes the inner product in $\mathbf{C}^{m}$.] This concludes the proof of $(2.1)$. Remark 5 follows from the same argument.

Proof of Corollary 1. Let $h \in L^{2}$ and $\max \left(1 / 2, p_{0}\right)<p \leq 1$. From Theorem 2 and the Hardy-Littlewood maximal theorem, it follows that

$$
c_{p}\|\mathbf{K} h\|_{H^{p}} \leq\|\mathbf{K} h\|_{L^{p}} \leq\|\mathbf{K} h\|_{H^{p}}
$$

where

$$
\|\mathbf{K} h\|_{H^{p}}=\left\|(\mathbf{K} h)^{+}\right\|_{L^{p}} .
$$

From the boundedness of singular integral operators on $H^{p}$, it follows that

$$
\|\mathbf{K} h\|_{H^{p}} \leq c_{p}\|h\|_{H^{p}} .
$$

On the other hand, by (1.1) there exist multipliers homogeneous of degree zero

$$
\Theta_{1}(\xi), \ldots, \Theta_{m}(\xi) \in C^{\infty}\left(S_{n-1}\right)
$$

such that

$$
\sum_{j=1}^{m} \theta_{j}(\xi) \Theta_{j}(\xi) \equiv 1 \quad \text { on } S_{n-1}
$$


So

(2.4) $\|h\|_{H^{p}}=\left\|\sum\left(\Theta_{j}(\xi)\left(K_{j} h\right)^{\wedge}(\xi)\right)^{\vee}\right\|_{H^{p}} \leq c_{p} \sum\left\|K_{j} h\right\|_{H^{p}} \leq c_{p}\|\mathbf{K} h\|_{H^{p}}$.

Thus, from (2.2)-(2.4), we get (1.7).

Let $h \in H^{p}$. Applying (1.7) to $h * P_{t}$, we get

$$
c_{1.4}\left\|h * P_{t}\right\|_{H^{p}} \leq \sum_{J=1}^{m}\left\|K_{j}\left(h * P_{t}\right)\right\|_{L^{p}} \leq c_{1.5}\left\|h * P_{t}\right\|_{H^{p}} .
$$

It is known that

$$
h * P_{t} \rightarrow h \quad \text { in } H^{p} \quad \text { as } t \rightarrow+0
$$

and that

$$
\sup _{t>0}\left|K_{J}\left(h * P_{t}\right)(x)\right| \in L^{p} .
$$

Thus by the Lebesgue dominated convergence theorem, we get

$$
\left\|K_{j}\left(h * P_{t}\right)\right\|_{L^{p}} \rightarrow\left(\int\left|\lim _{t \rightarrow+0} K_{j}\left(h * P_{t}\right)(x)\right|^{p} d x\right)^{1 / p} \text { as } t \rightarrow+0 .
$$

Therefore, letting $t \rightarrow+0$ in (2.5), we get (1.8).

LEMMA 2.1. Let $u(x, t)$ be a nonnegative function defined on $\mathbf{R}^{n} \times$ $[0,+\infty)$ and continuous on $\mathbf{R}^{n} \times(0,+\infty)$. Let $q>1$. If

$$
u(x, 0)=\lim _{t \rightarrow+0} u(x, t) \text { a.e. } x
$$

and if

$$
\left|\left\{x \in \mathbf{R}^{n}: \sup _{t \geq 0} u(x, t)>\lambda\right\}\right| \leq \lambda^{-q}
$$

for any $\lambda>0$, then

$$
\lim _{t \rightarrow+0} M_{1}(u(\cdot, t))(x)=M_{1}(u(\cdot, 0))(x) \text { a.e. } x .
$$

Proof. Take any $\varepsilon>0$. By (2.6) and (2.7) there exists $t_{0}>0$ such that $|G|<\varepsilon$, where

$$
G=\left\{x \in \mathbf{R}^{n}: \sup _{t \in\left[0, t_{0}\right]}|u(x, t)-u(x, 0)|>\varepsilon\right\} .
$$

Since

$$
\int_{G} \sup _{t \geq 0} u(x, t) d x<C \varepsilon^{1-1 / q}
$$


by (2.7), there exists a measurable set $E$ such that

$$
\begin{aligned}
& |E|<C \varepsilon^{(1-1 / q) / 2}, \\
& \left|M_{1}(u(\cdot, t))(x)-M_{1}(u(\cdot, 0))(x)\right|<C \varepsilon^{(1-1 / q) / 2}+\varepsilon
\end{aligned}
$$

for any $x \in E^{c}$ and any $t \in\left[0, t_{0}\right]$. Since $\varepsilon>0$ is arbitrary, we get (2.8).

Proof of Corollary 2. Put $\theta_{0} \equiv 1$ and $K_{0}=$ the identity operator. By the usual argument about maximal singular integral operators and the Hardy-Littlewood maximal theorem, we get

$$
\lambda\left|\left\{x \in \mathbf{R}^{n}: \sum_{j=0}^{m} \sup _{t>0}\left|K_{j}\left(h * P_{t}\right)(x)\right|>\lambda\right\}\right| \leq C\|h\|_{M}
$$

for any $\lambda>0$ and

(2.9) $\limsup _{\lambda \rightarrow+\infty} \lambda\left|\left\{x \in \mathbf{R}^{n}: \sum_{j=0}^{m} \sup _{t>0}\left|K_{j}\left(h * P_{t}\right)(x)\right|>\lambda\right\}\right| \leq C\|s\|_{M}$.

It is also known that

$$
\kappa_{j}(x)=\lim _{t \rightarrow+0} K_{j}\left(h * P_{t}\right)(x)
$$

exists almost everywhere and that $\kappa_{0}(x)=f(x)$ a.e. By (2.9)

(2.10) $\limsup _{\lambda \rightarrow+\infty} \lambda\left|\left\{x \in \mathbf{R}^{n}: M_{1 / 2}\left(\sum_{j=0}^{m}\left|\kappa_{j}\right|\right)(x)>\lambda\right\}\right| \leq C\|s\|_{M}$.

Applying Lemma 2.1 to

$$
u(x, t)= \begin{cases}\left(\sum_{j=0}^{m}\left|K_{j}\left(h * P_{t}\right)(x)\right|\right)^{1 / 2} & \text { if } t>0, \\ \left(\sum_{j=0}^{m}\left|\kappa_{j}(x)\right|\right)^{1 / 2} & \text { if } t=0,\end{cases}
$$

and $q=2$, we get

$$
M_{1 / 2}\left(\sum_{J=0}^{m}\left|K_{j}\left(h * P_{t}\right)\right|\right)(x) \rightarrow M_{1 / 2}\left(\sum_{j=0}^{m}\left|\kappa_{j}\right|\right)(x) \quad \text { a.e. } x \quad \text { as } t \rightarrow+0 \text {. }
$$

Similarly

(2.11) $M_{p_{0}}\left(M_{1 / 2}\left(\sum_{j=0}^{m}\left|K_{j}\left(h * P_{t}\right)\right|\right)\right)(x) \rightarrow M_{p_{0}}\left(M_{1 / 2}\left(\sum_{j=0}^{m}\left|\kappa_{j}\right|\right)\right)(x)$ 
Since $\theta_{1}, \ldots, \theta_{m}$ satisfy $(1.9), \theta_{0}, \ldots, \theta_{m}$ satisfy (1.1). By Remark 5 ,

$$
\begin{aligned}
\left(h * P_{t}\right)^{++}(x) & =\left(K_{0}\left(h * P_{t}\right)\right)^{++}(x) \\
& \leq C M_{p_{0}}\left(M_{1 / 2}\left(\sum_{J=0}^{m}\left|K_{j}\left(h * P_{t}\right)\right|\right)\right)(x)
\end{aligned}
$$

for any $t>0$. Letting $t \rightarrow+0$, we get

$$
h^{++}(x) \leq C M_{p_{0}}\left(M_{1 / 2}\left(\sum\left|\kappa_{j}\right|\right)\right)(x) \text { a.e. } x
$$

form (2.11).

On the other hand, [18] and [34] showed

$$
\liminf _{\lambda \rightarrow+\infty} \lambda\left|\left\{x \in \mathbf{R}^{n}: h^{++}(x)>\lambda\right\}\right| \geq c\|s\|_{M},
$$

where $c>0$ depends only on the dimension. Thus, for a sufficient large $\lambda$, we have

$$
\begin{aligned}
\|s\|_{M} & \leq C \lambda\left|\left\{x \in \mathbf{R}^{n}: M_{p_{0}}\left(M_{1 / 2}\left(\sum\left|\kappa_{j}\right|\right)\right)(x)>\lambda\right\}\right| \\
& \leq C \lambda^{1-p_{0}} \int_{\left\{M_{1 / 2}\left(\sum \mid \kappa_{j}\right)(x)>\lambda / 2\right\}} M_{1 / 2}\left(\sum\left|\kappa_{j}\right|\right)(x)^{p_{0}} d x \\
& \leq C \lambda^{1-p_{0}}\left|\left\{M_{1 / 2}\left(\sum\left|\kappa_{j}\right|\right)(x)>\lambda / 2\right\}\right|^{1-p_{0}}\|s\|_{M}^{p_{0}}
\end{aligned}
$$

by (2.10). Therefore

$$
\lambda\left|\left\{M_{1 / 2}\left(\sum\left|\kappa_{j}\right|\right)(x)>\lambda\right\}\right| \geq C\|s\|_{M} \text { as } \lambda \rightarrow+\infty .
$$

Repeating the same argument, we get

$$
\lambda\left|\left\{\sum_{J=0}^{m}\left|\kappa_{j}(x)\right|>\lambda\right\}\right| \geq C\|s\|_{M} \quad \text { as } \lambda \rightarrow+\infty .
$$

Since $\lambda\left\{\left|\kappa_{0}(x)\right|>\lambda\right\} \mid \rightarrow 0$ as $\lambda \rightarrow+\infty$, we get Corollary 2 .

3. Jones's formula. In this section, we explain the relation between Theorem 1 and Jones's recent work [25].

Definition 3.A. A complex measure on the upper half-plane $\mathbf{R}_{+}^{2}=$ $\{(x, t): x \in \mathbf{R}, t>0\}$ is called a Carleson measure if

$$
\sup _{I}|\mu|(Q(I)) /|I|=\|\mu\|_{c}<+\infty,
$$

where $|\mu|$ is the total variation of $\mu, I$ is taken over all intervals. 
Suppose that $\|\mu\|_{c} \leq 1$. It has been shown by Carleson [3] [see also Hörmander [19]] that there exists $F \in L^{\infty}(\mathbf{R})$ such that

$$
\|F\|_{L^{\infty}} \leq C
$$

and such that

$$
\int_{R} f(x) F(x) d x=\iint_{R_{+}^{2}} f(x, t) d \mu(x, t)
$$

for any $f \in L^{1}(\mathbf{R})$ with $\operatorname{supp} \hat{f} \subset[0,+\infty)$, where

$$
\begin{aligned}
f(x, t) & =\int_{R} P_{t}(y) f(x-y) d y, \\
P_{t}(y) & =t /\left(\pi\left(y^{2}+t^{2}\right)\right) .
\end{aligned}
$$

Recently, Jones [25] gave an explicit formula for the construction of $F$.

Definition 3.B. [Jones [25].] For a measure $\mu$ on $\mathbf{R}_{+}^{2}$ let

$$
\begin{aligned}
J(\mu, x, \zeta)= & \frac{1}{\pi} \frac{\operatorname{Im} \zeta}{(x-\zeta)(x-\bar{\zeta})} \\
& \times \exp \left(\int_{0<\operatorname{Im} \eta \leq \operatorname{Im} \zeta} \frac{-i}{x-\bar{\eta}}+\frac{i}{\zeta-\bar{\eta}} d|\mu|(\eta)\right)
\end{aligned}
$$

where $i=(-1)^{1 / 2}$, and $\zeta$ and $\eta$ are complex numbers. [We identify $\eta$ with $(\operatorname{Re} \eta, \operatorname{Im} \eta) \in \mathbf{R}^{2}$.]

THEOREM 3.A. [ Jones [25].] Let $\|\mu\|_{c} \leq 1$. Set

$$
F(x)=\iint_{R_{+}^{2}} J(\mu, x, \zeta) d \mu(\zeta)
$$

Then,

$$
\|F\|_{L^{\infty}} \leq C
$$

and (3.2) holds.

Our Theorem 1 can be regarded as a generalization of the formula (3.3). In Jones's argument, we can replace the formula (3.3) by Theorem 1. In the following, we sketch it.

Let $H$ be the Hilbert transform, that is

$$
H f=(-i(\operatorname{sign} \xi) \hat{f}(\xi))^{2}
$$


For $t>0$ set

$$
u_{t}(x)=\iint_{\substack{0 \leq s \leq t, j \\ y \in \mathbb{R}}} s^{-1}(1+|x-y| / s)^{-3 / 2} d|\mu|(y, s) .
$$

LEMMA 3.A. [See [9] and [22].] Let $\|\mu\|_{c} \leq 1$. Then,

$$
\left\|u_{t}\right\|_{\mathrm{BLO}} \leq C_{3.1}
$$

and

$$
\int_{I} u_{t} d x \leq C_{3.1}|I|
$$

for any cube $I$ with $l(I) \geq t$.

Set $\varepsilon=c_{0} / C_{3.1}$. Then $e^{-\varepsilon u_{t}(x)}$ satisfies (1.4) and

$$
\int_{y-t}^{y+t} e^{-\varepsilon u_{t}(x)} d x / t \geq C
$$

for any $y \in \mathbf{R}$. So, by applying Theorem 1 and Remark 3 to $K_{1}=$ the identity operator and $K_{2}=-H$ and by using dilation and translation, for each $(y, t) \in \mathbf{R}_{+}^{2}$ we get real-valued functions $g_{1,(y, t)}(x)$ and $g_{2,(y, t)}(x)$ such that

$$
\begin{gathered}
P_{t}(y-x)-g_{1,(y, t)}(x)-H g_{2,(y, t)}(x) \equiv 0, \\
\left|g_{j,(y, t)}(x)\right| \leq C e^{-\varepsilon u_{t}(x)} t^{-1}(1+|y-x| / t)^{-3 / 2} \quad(j=1,2) .
\end{gathered}
$$

Set

$$
F(x)=\iint_{R_{+}^{2}} g_{1,(y, t)}(x)+i g_{2,(y, t)}(x) d \mu(y, t) .
$$

Then

$$
\begin{aligned}
& |F(x)| \leq C \iint_{R_{+}^{2}} e^{-\varepsilon u_{t}(x)} t^{-1}(1+|y-x| / t)^{-3 / 2} d|\mu|(y, t) \\
& =C \iint \exp \left(-\varepsilon \iint_{\substack{0<s \leq t, v \in R}} s^{-1}(1+|x-v| / s)^{-3 / 2} d|\mu|(v, s)\right) \\
& \cdot t^{-1}(1+|y-x| / t)^{-3 / 2} d|\mu|(y, t) \\
& \leq C \varepsilon^{-1}\left[\exp \left(-\varepsilon \iint_{\substack{0<s \leq t, v \in \mathcal{R}}} s^{-1}(1+|x-v| / s)^{-3 / 2} d|\mu|(v, s)\right)\right]_{t=+\infty}^{t=0} \\
& \leq C \varepsilon^{-1}
\end{aligned}
$$


and

$$
\begin{aligned}
\int f(x) F(x) d x & =\iint_{R_{+}^{2}} d \mu(y, t) \int\left(g_{1,(y, t)}(x)+i g_{2,(y, t)}(x)\right) f(x) d x \\
& =\iint_{R_{+}^{2}} d \mu(y, t) \int P_{t}(y-x) f(x) d x=\iint_{R_{+}^{2}} f(y, t) d \mu(y, t)
\end{aligned}
$$

for any $f \in L^{1}(\mathbf{R})$ with supp $\hat{f} \subset[0,+\infty)$.

4. Weighted BMO. In the following, we assume (1.4).

Definition 4.1. For a measurable set $E$ let

$$
m_{w}(E)=\int_{E} w(x) d x
$$

and

$$
w(E)=\sup _{x \in E} w(x)
$$

Definition 4.2. For $\mathbf{f}(x) \in L_{\mathrm{loc}}^{1}\left(\mathbf{R}^{n}, \mathbf{C}^{m}\right)$, let

$$
\|\mathbf{f}\|_{\mathrm{BMO} w}=\sup _{I} \int_{I}\left|\mathbf{f}(x)-\mathbf{f}_{I}\right| d x / m_{w}(I),
$$

where the supremum is taken over all cubes in $\mathbf{R}^{n}$ and $\mathbf{f}_{I}=\int_{I} \mathbf{f} d x /|I|$.

For the scalar-valued case, this definition is due to MuckenhouptWheeden [26]-[27].

We prepare some easy lemmas.

LEMMA 4.A. If $\|\mathbf{f}\|_{\mathrm{BMO}{ }_{w}} \leq 1$, then for any cube $I$ and any $\lambda>0$,

$$
\left|\left\{x \in I:\left|\mathbf{f}(x)-\mathbf{f}_{I}\right|>\lambda\right\}\right| /|I| \leq C_{4.1} e^{-C_{4.2} \lambda / w(I)} .
$$

LEMMA 4.B. For any cube $I$ and any $\lambda>0$

$$
|\{x \in I:-\log w(x)>-\log w(I)+\lambda\}| /|I| \leq C_{4.1} e^{-C_{4.2} \lambda / c_{0}} .
$$

These follow from [21], (1.4) and [9].

LEMMA 4.1. For any cubes $I$ and $J$ and for any $t>0$,

$$
|\{x \in I: w(x) \leq t w(I)\}| /|I| \leq C_{4.1} t^{C_{42} / c_{0}},
$$


(4.2) $\int_{I} w(I)-w(x) d x / m_{w}(I) \leq C c_{0}$ i.e. $\left(1+C c_{0}\right) m_{w}(I) \geq w(I)|I|$, if $J \supset I$, then $w(J) / w(I) \leq C(|J| /|I|)^{c_{0} / C_{4.2}}$,

(4.4) if $|I|=|J|$, then $w(J) / w(I) \leq C\left(1+\left|x_{I}-x_{J}\right| / l(I)\right)^{c_{0} n / C_{4.2}}$.

LEMMA 4.2.

$$
\|w\|_{\text {BMO } w} \leq C c_{0} .
$$

The above two lemmas are easy consequences of Lemma 4.B.

DeFINITION 4.3. For $0<\varepsilon \leq 1$, let

$$
\begin{aligned}
\|\mathbf{f}\|_{\text {Lip } \varepsilon} & =\sup _{x, y: x \neq y}|\mathbf{f}(x)-\mathbf{f}(y)| /|x-y|^{\varepsilon}, \\
\|\mathbf{f}\|_{\text {Lip } 2} & =\sum_{J=1}^{n}\left\|\frac{\partial}{\partial x_{j}} \mathbf{f}\right\|_{\text {Lip 1 }} .
\end{aligned}
$$

LEMMA 4.3. If $1 \geq \varepsilon \geq c_{0} n / C_{4.2}$ and if $\operatorname{supp} \subset I(0, t)$, then

$$
\|\mathbf{f}\|_{\mathrm{BMO}_{w}} \leq C t^{\varepsilon}\|\mathbf{f}\|_{\text {Lip } \varepsilon} / w(I(0, t)) .
$$

Proof. We may assume $t=1$. Take any cube $I$ in $\mathbf{R}^{n}$. If $l(I)>1$ and $I \cap I(0,1) \neq \varnothing$, then

$$
\int_{I}\left|\mathbf{f}(x)-\mathbf{f}_{I}\right| d x / m_{w}(I) \leq C\|\mathbf{f}\|_{L^{1} / m_{w}}(I) \leq C\|\mathbf{f}\|_{\operatorname{Lip} \varepsilon} / w(I(0,1)) .
$$

If $l(I) \leq 1$ and $I \cap I(0,1) \neq \varnothing$, then

$$
\int_{I}\left|\mathbf{f}(x)-\mathbf{f}_{I}\right| d x / m_{w}(I) \leq C l(I)^{\varepsilon}\|\mathbf{f}\|_{\operatorname{Lip} \varepsilon} / w(I) \leq C\|\mathbf{f}\|_{\operatorname{Lip} \varepsilon} / w(I(0,1))
$$

by (4.3).

MAIN Lemma. Let $c_{0}>0$ be small enough depending only on $\theta_{1}, \ldots, \theta_{m}$. Let $t>0$. Suppose that (1.4),

$$
\|\mathbf{f}\|_{\mathrm{BMO} w}<c_{0}
$$

and

$$
\operatorname{supp} \mathbf{f} \subset I(0, t)
$$


hold. Then there exists $\mathbf{g}(x)$ such that

$$
|\mathbf{g}(x)| \leq w(x)(1+|x| / t)^{-n-1 / 2}
$$

and

$$
\mathbf{f}-\mathbf{g} \in S \text {. }
$$

We prove this Main Lemma in $\$ 9$.

5. Proof of Theorem 1. Let $h(t) \in C^{\infty}([0,+\infty))$ be such that

$$
h(t) \geq 0, \quad \operatorname{supp} h \subset[1 / 4,1],
$$

and

$$
\sum_{k=1}^{\infty} h_{k}(t)=1 \text { on }[1,+\infty)
$$

where

$$
h_{k}(t)=h\left(2^{-k} t\right) \quad \text { for } k=1,2,3, \ldots
$$

Set

$$
h_{0}(t)=1-\sum_{k=1}^{\infty} h_{k}(t) \text {. }
$$

Then

$$
\mathbf{f}(x)=\sum_{k=0}^{\infty} h_{k}(|x|) \mathbf{f}(x)
$$

and

$$
\left\|h_{k}(|x|) \mathbf{f}(x)\right\|_{\mathrm{BMO}_{w}} \leq C 2^{k}\left\|h_{k} \mathbf{f}\right\|_{\text {Lip } 1} / w\left(I\left(0,2^{k}\right)\right) \leq C 2^{-k(n+1)} / w(I(0,1))
$$

by (1.2), (1.3) and Lemma 4.3.

Applying the Main Lemma in $\S 4$ to each $h_{k} \mathbf{f}$, we get $\mathbf{g}_{k}$ such that

$$
\begin{gathered}
h_{k} \mathbf{f}-\mathbf{g}_{k} \in S, \\
\left|\mathbf{g}_{k}(x)\right| \leq c_{0}^{-1} C 2^{-k(n+1)} w(x)\left(1+2^{-k}|x|\right)^{-n-1 / 2} / w(I(0,1)) .
\end{gathered}
$$

Set

$$
\mathbf{g}(x)=\sum_{k=0}^{\infty} \mathbf{g}_{k}(x)
$$

Then (1.5) is clear and (1.6) follows from

$$
\begin{aligned}
\sum_{k=0}^{\infty}\left|\mathbf{g}_{k}(x)\right| & \leq c_{0}^{-1} C w(x) \sum 2^{-k(n+1)}\left(1+2^{-k}|x|\right)^{-n-1 / 2} / w(I(0,1)) \\
& \leq c_{0}^{-1} C w(x)(1+|x|)^{-n-1 / 2} / w(I(0,1)) .
\end{aligned}
$$


6. The property of the space $S$. The hard part in our argument is the problem, "What property does the space $S$ have?" Since $\theta_{1}, \ldots, \theta_{m}$ satisfy $(1.1), \bar{\theta}_{1}, \ldots, \bar{\theta}_{m}$ satisfy (1.1). Then by Lemma 2.2 of [32] there exist functions

$$
\Theta_{j}(\xi, \nu) \in C^{\infty}\left(S_{n-1} \times \sum_{2 m-1}\right), \quad 1 \leq j \leq m
$$

such that

$$
\begin{aligned}
& \sum_{j=1}^{m} \bar{\theta}_{j}(\xi) \Theta_{j}(\xi, \boldsymbol{\nu}) \equiv 1, \\
& \operatorname{Re} \sum_{j=1}^{m} \bar{\nu}_{j}\left(\Theta_{j}(\xi, \nu)+\Theta_{j}(-\xi, \nu)\right) \equiv 0, \\
& \operatorname{Im} \sum_{j=1}^{m} \bar{\nu}_{j}\left(\Theta_{j}(\xi, \nu)-\Theta_{j}(-\xi, \nu)\right) \equiv 0 .
\end{aligned}
$$

This fact tells us that for any $\nu \in \Sigma_{2 m-1}$ the set of real-valued functions

$$
\{\langle\mathbf{p}(x), \boldsymbol{\nu}\rangle: \mathbf{p} \in S\}
$$

is a sufficiently large class of functions. More precisely, we obtain

LemMa 6.1. Let $\boldsymbol{\nu} \in \Sigma_{2 m-1}$. Let I be a cube. Let $b(x)$ be a real-valued function such that

$$
\begin{aligned}
& \text { supp } b \subset 3 I, \\
& \int b(x) d x=0, \\
& \|b\|_{\text {Lip } 2} \leq l(I)^{-2} .
\end{aligned}
$$

Then there exists a $\mathbf{C}^{m}$-valued function $\mathbf{p}(x)$ such that

(6.4) $\mathbf{p} \in S$,

(6.5) $\int \mathbf{p}(x) d x=0$,

(6.6) $\langle\mathbf{p}(x), \boldsymbol{\nu}\rangle \equiv b(x)$,

(6.7) $|\mathbf{p}(x)| \leq C\left(1+\left|x-x_{I}\right| / l(I)\right)^{-n-1}$, '

$$
\left|\frac{\partial}{\partial x_{j}} \mathbf{p}(x)\right| \leq C l(I)^{-1}\left(1+\left|x-x_{I}\right| / l(I)\right)^{-n-2}, \quad j=1, \ldots, n .
$$

Proof. Set

$$
\begin{aligned}
& \tilde{p}_{j}(x)=-\left(\Theta_{j}(\xi, \boldsymbol{\nu})\left(\operatorname{Re}\left(\mathbf{K}^{*} \cdot(b \boldsymbol{v})\right)\right)^{\hat{\imath}}(\xi)\right)^{\swarrow}(x) \\
& -i\left(\Theta_{j}(\xi, i \boldsymbol{\nu})\left(\operatorname{Im}\left(\mathbf{K}^{*} \cdot(b \boldsymbol{v})\right)\right)^{\hat{(}}(\xi)\right)^{\llcorner}(x)
\end{aligned}
$$


and

$$
\tilde{\mathbf{p}}(x)=\left(\tilde{p}_{1}(x), \ldots, \tilde{p}_{m}(x)\right) .
$$

By the properties of $\left\{\Theta_{j}\right\}$ and by the same argument as in Lemma 2.3 of [32],

$$
\mathbf{K}^{*} \cdot \tilde{\mathbf{p}}=-\mathbf{K}^{*} \cdot(b \boldsymbol{v}), \quad \int \tilde{\mathbf{p}}(x) d x=0, \quad\langle\tilde{\mathbf{p}}(x), \boldsymbol{\nu}\rangle \equiv 0 .
$$

Set

$$
\mathbf{p}(x)=\tilde{\mathbf{p}}(x)+b(x) \boldsymbol{\nu} .
$$

Then (6.4)-(6.6) hold. Since $\tilde{p}_{j}(x)$ can be written in the form of a linear combination of $b$ and its images by Calderon-Zygmund singular integral operators with smooth kernels [see Stein [29] p. 75], (6.7)-(6.8) follow from (6.1)-(6.3). See Lemma 2.3 of [32] for details.

Lemma 6.2. The function $\mathbf{p}(x)$ of Lemma 6.1 can be decomposed as follows:

$$
\begin{gathered}
\mathbf{p}(x)=\sum_{j=4}^{\infty} 2^{-\jmath(n+1)} \boldsymbol{\beta}_{j}(x), \quad \operatorname{supp} \boldsymbol{\beta}_{J} \subset 2^{j} I, \\
\left\|\boldsymbol{\beta}_{J}\right\|_{\text {Lip } 1} \leq C /\left(2^{\prime} l(I)\right), \\
\int \boldsymbol{\beta}_{j}(x) d x=0, \\
\left\langle\boldsymbol{\beta}_{j}(x), \boldsymbol{\nu}\right\rangle \equiv 0 \quad \text { if } j>4, \quad\left\langle\boldsymbol{\beta}_{4}(x), \boldsymbol{\nu}\right\rangle \equiv b(x) .
\end{gathered}
$$

Proof. Let $h_{k}(x)$ be as in (5.2)-(5.3). Then

$$
\begin{aligned}
\mathbf{p}(x)= & \left\{h_{0}\left(2^{-4}|x|\right) \mathbf{p}(x)+h_{4}(|x|) \int \sum_{k=5}^{\infty} h_{k}(|y|) \mathbf{p}(y) d y / \int h_{4}(|y|) d y\right\} \\
& +\sum_{j=5}^{\infty}\left\{h_{j}(|x|) \mathbf{p}(x)-h_{J-1}(|x|) \int \sum_{k=j}^{\infty} h_{k}(|y|) \mathbf{p}(y) d y / \int h_{,-1}(|y|) d y\right. \\
& \left.+h_{J}(|x|) \int \sum_{k=j+1}^{\infty} h_{k}(|y|) \mathbf{p}(y) d y / \int h_{j}(|y|) d y\right\}
\end{aligned}
$$

gives the desired decomposition. See Lemma 3.5 of [32] for details. 
7. Weighted Carleson measures. We continue to assume (1.4).

Definition 7.1. For a measure $\mu$ defined on $\mathbf{R}_{+}^{n+1}$, let

$$
\|\mu\|_{c, w}=\sup _{I}|\mu|(Q(I)) / m_{w}(I),
$$

where $I$ is taken over all cubes in $\mathbf{R}^{n}$.

We prepare some easy lemmas.

LEMMA 7.1. If $\|\mu\|_{c, w} \leq 1$, then for any cube I

$$
\iint_{Q(I)} w(I(x, t))^{-1} d|\mu|(x, t) \leq C|I| .
$$

Proof. [For the definition of $w(I(x, t))$ recall Definition 4.1.] We may assume that $I$ is a closed dyadic cube. Let $\left\{I_{k, j}\right\}_{j=1}^{\infty}$ be the maximal closed dyadic subcubes of $I$ such that

$$
w\left(I_{k, J}\right) \leq 2^{-k} w(I) .
$$

By (4.1)

$$
\sum_{j}\left|I_{k, j}\right| \leq C_{4.1} 2^{-C_{42} k / c_{0}}|I|
$$

So

$$
\begin{aligned}
\iint_{Q(I)} w(I(x, t))^{-1} d|\mu| & \leq C \sum_{k=0}^{\infty} \sum_{j} \iint_{Q\left(I_{k, j}\right)} 2^{k+1} w(I)^{-1} d|\mu| \\
& \leq C w(I)^{-1} \sum_{k} \sum_{j} 2^{k+1} m_{w}\left(I_{k, j}\right) \\
& \leq C w(I)^{-1} \sum_{k} 2^{k+1} \sum_{j} 2^{-k} w(I)\left|I_{k, j}\right| \\
& \leq C \sum_{k} C_{4.1} 2^{-C_{4.2} k / c_{0}}|I| \leq C|I| .
\end{aligned}
$$

DEFINITION 7.2. For nonnegative real numbers $\left\{\lambda_{I}\right\}_{I}$, where $I$ is taken over all dyadic cubes, set

$$
\begin{aligned}
& \eta_{k}(x)=\sum_{I: l(I)=2^{-k}} \lambda_{I}\left(1+2^{k}\left|x-x_{I}\right|\right)^{-n-1}, \\
& \varepsilon_{k}(x)=\sum_{j=0}^{\infty}\left(\frac{2}{3}\right)^{j} \eta_{k-j}(x) .
\end{aligned}
$$


LEMMA 7.2.

$$
\begin{gathered}
\lambda_{I} \leq\left(1+2^{k}\left|x-x_{I}\right|\right)^{n+1} \eta_{k}(x) \quad \text { if } l(I)=2^{-k}, \\
\eta_{k}(x) \leq\left(1+2^{k}|x-y|\right)^{n+1} \eta_{k}(y), \\
\varepsilon_{k}(x) \leq\left(1+2^{k}|x-y|\right)^{n+1} \varepsilon_{k}(y) .
\end{gathered}
$$

Since this is easy, we omit the proof.

LEMMA 7.3. Let $c_{0}>0$ be small enough in (1.4). Let

$$
\left\|\sum \lambda_{I}^{2}|I| \delta_{\left(x_{I}, l(I)\right)}\right\|_{c, w^{2}} \leq 1 .
$$

Then

$$
\begin{gathered}
\eta_{k}(x) \leq \varepsilon_{k}(x) \leq C w\left(I\left(x, 2^{-k}\right)\right), \\
\left\|\sum_{k=-\infty}^{+\infty} \varepsilon_{k}(x)^{2} \delta_{t=2^{-k}}\right\|_{c, w^{2}} \leq C,
\end{gathered}
$$

where $\delta_{(x, t)}$ is the Dirac measure concentrated at the point $(x, t) \in \mathbf{R}_{+}^{n+1}$ and $\delta_{t=a}$ denotes the measure induced from $n$-dimensional Lebesgue measure on the hyperplane $t=a$ in $\mathbf{R}_{+}^{n+1}$.

$$
\begin{aligned}
& \text { Proof. Since } \lambda_{J} \leq C w(J) \\
& \qquad \begin{aligned}
\eta_{k}(x) & \leq C \sum_{J: l(J)=2^{-k}}\left(1+2^{k} \operatorname{dist}(x, J)\right)^{-n-1} w(J) \\
& \leq C \sum(\cdots)^{-n-1+C c_{0}} w\left(I\left(x, 2^{-k}\right)\right) \quad \text { by }(4.4) \\
& \leq C w\left(I\left(x, 2^{-k}\right)\right)
\end{aligned}
\end{aligned}
$$

So,

$$
\begin{aligned}
\varepsilon_{k}(x) & \leq C \sum_{j=0}^{\infty}\left(\frac{2}{3}\right)^{j} w\left(I\left(x, 2^{-k+\jmath}\right)\right) \\
& \leq C \sum_{j=0}^{\infty}\left(\frac{2}{3}\right)^{j} 2^{j c_{0} C} w\left(I\left(x, 2^{-k}\right)\right) \quad \text { by }(4.3) \\
& \leq C w\left(I\left(x, 2^{-k}\right)\right) .
\end{aligned}
$$

Condition (7.6) follows from almost the same argument as Lemma 3.2 of [32] with slight additional estimates about the order of growth of $w$ as in the proof of (7.5). We omit the proof. 
8. The decomposition of weighted BMO functions. We continue to assume (1.4).

Following Chang-R. Fefferman [7], we decompose a weighted BMO function $\mathbf{f}(x)$ and the weight function $w(x)$.

LEMMA 8.1. Suppose that $\operatorname{supp} \mathbf{f} \subset I(0,1)$ and $\|\mathbf{f}\|_{\mathrm{BMO} w} \leq 1$. Then there exist $\mathbf{C}^{m}$-valued functions $\left\{\mathbf{b}_{I}(x)\right\}_{I}$ and nonnegative real numbers $\left\{\lambda_{I}\right\}_{I}$, where $I$ is taken over all dyadic cubes in $\mathbf{R}^{n}$, such that

$$
\begin{gathered}
\mathbf{f}=\sum_{I} \lambda_{I} \mathbf{b}_{I}, \\
\lambda_{I}=0 \quad \text { if } 3 I \cap I(0,1)=\varnothing, \\
\operatorname{supp} \mathbf{b}_{I} \subset 3 I, \\
\int \mathbf{b}_{I} d x=0, \\
\left\|\mathbf{b}_{I}\right\|_{\text {Lip } 2} \leq C l(I)^{-2}, \\
\left\|\sum_{I} \lambda_{I}^{2}|I| \delta_{\left(x_{I}, I(I)\right)}\right\|_{c, w^{2}} \leq C .
\end{gathered}
$$

Proof. We use the idea of Chang-R. Fefferman [7]. Take a real-valued function $\varphi(x) \in \mathscr{Q}\left(\mathbf{R}^{n}\right)$ such that

$$
\begin{gathered}
\operatorname{supp} \varphi \subset\left\{x \in \mathbf{R}^{n}:|x|<1\right\}, \\
\int_{0}^{+\infty} \hat{\varphi}(\xi t)^{2} t^{-1} d t \equiv 1 \text { for any } \xi \in \mathbf{R}^{n} \backslash\{0\}
\end{gathered}
$$

Set

$$
\lambda_{I}=|I|^{-1 / 2}\left(\iint_{T(I)}\left|\varphi_{t} * \mathbf{f}(y)\right|^{2} t^{-1} d t d y\right)^{1 / 2}
$$

and

$$
\mathbf{b}_{I}(x)=\iint_{T(I)} \varphi_{t}(x-y)\left(\varphi_{t} * \mathbf{f}\right)(y) t^{-1} d t d y / \lambda_{I},
$$

where we define $0 / 0=0$ and

$$
T(I)=\{(x, t): x \in I, t \in(l(I) / 2, l(I))\} .
$$

Then (8.2) is clear. Conditions (8.3)-(8.5) follow from the same argument as in Lemma 3.1 and Remark 3.1 of [32]. See [32] for details. 
Since

$$
\begin{aligned}
\sum_{I \subset J} \lambda_{I}^{2}|I| & =\iint_{Q(J)}\left|\varphi_{t} * \mathbf{f}(y)\right|^{2} t^{-1} d t d y \\
& \leq C \iint_{3 J}\left|\mathbf{f}(x)-\mathbf{f}_{J}\right|^{2} d x \leq C|J| w(J)^{2}
\end{aligned}
$$

for any dyadic cube $J$ by Lemma 4.A, (8.6) holds.

LEMMA 8.2. Let $k>0$. In Lemma 8.1 set

$$
\mathbf{f}_{k}(x)=\sum_{I: l(I) \geq 2^{-k}} \lambda_{I} \mathbf{b}_{I}(x)
$$

Then

$$
\begin{gathered}
\operatorname{supp} \mathbf{f}_{k} \subset I(0,3) \\
\left|\mathbf{f}_{k}(x)-\mathbf{f}_{k}(y)\right| \leq C w\left(I\left(x, 2^{-k}\right)\right) 2^{k}|x-y| \\
\text { provided }|x-y|<2^{-k}
\end{gathered}
$$

Proof. Set

$$
\begin{aligned}
\Phi & =\left(\int_{1}^{\infty} \hat{\varphi}(t \xi)^{2} t^{-1} d t\right)^{-} \\
& =\lim _{\varepsilon \rightarrow+0 \text { in } \varsigma^{\prime}}\left(\delta_{0}-\int_{\varepsilon}^{1} \varphi_{t} * \varphi_{t} t^{-1} d t\right),
\end{aligned}
$$

where $\delta_{0}$ is the dirac measure concentrated at the origin. Since

$$
\mathbf{f}_{k}=\mathbf{f} * 2^{k n} \boldsymbol{\Phi}\left(2^{k} \cdot\right),
$$

(8.7) is clear. (8.8) follows from $\|\mathbf{f}\|_{\mathrm{BMO}{ }_{w}} \leq 1$ and from

$$
\mathbf{f}_{k}(x)-\mathbf{f}_{k}(y)=\int \mathbf{f}(z) 2^{k n}\left(\Phi\left(2^{k}(x-z)\right)-\Phi\left(2^{k}(y-z)\right)\right) d z .
$$

From Lemmas 8.1-8.2 we get

LEMMA 8.3. Let $\|\mathbf{f}\|_{\mathrm{BMO} w} \leq c_{0}$. Let supp $\mathbf{f} \subset I(0,1)$. Let $M$ be $a$ positive integer. Then there exist $\mathbf{f}_{M}(x),\left\{\mathbf{b}_{I}(x)\right\}_{I \text { : dyadic }}$ and nonnegative real numbers $\left\{\lambda_{f, I}\right\}_{I \text { : dyadic }}$ such that

$$
\mathbf{f}=\sum_{I} \lambda_{f, I} \mathbf{b}_{I}+\mathbf{f}_{M}
$$


$(8.10)$

$$
\lambda_{f, I}=0 \quad \text { if } 3 I \cap I(0,1)=\varnothing \text { or if } l(I) \geq 2^{-M},
$$

$(8.3)-(8.5)$,

$$
\left\|\sum_{I} \lambda_{f, I}^{2}|I| \delta_{\left(x_{I}, l(I)\right)}\right\|_{c, w^{2}} \leq C c_{0}^{2}
$$

$$
\operatorname{supp} \mathbf{f}_{M} \subset I(0,3),
$$

$$
\left|\mathbf{f}_{M}(x)-\mathbf{f}_{M}(y)\right| \leq C c_{0} w\left(I\left(x, 2^{-M}\right)\right) 2^{M}|x-y|
$$

provided $|x-y|<2^{-M}$.

LEMMA 8.4. Let $c_{0}>0$ be small enough in (1.4). Let $M$ be a positive integer. Then there exist real-valued functions $w_{M}(x),\left\{b_{I}(x)\right\}_{I \text { : dyadic }}$ and nonnegative real numbers $\left\{\lambda_{w, I}\right\}_{I \text { : dyadic }}$ such that

$$
\begin{aligned}
& w=\sum_{I} \lambda_{w, I} b_{I}+w_{M}, \\
& \lambda_{w, I}=0 \text { if } l(I) \geq 2^{-M}, \\
& \operatorname{supp} b_{I} \subset 3 I, \\
& \int b_{I} d x=0, \\
& \left\|b_{I}\right\|_{\text {Lip } 2} \leq C l(I)^{-2}, \\
& \left\|\sum_{I} \lambda_{w, I}^{2} I \mid \delta_{\left(x_{I}, l(I)\right)}\right\|_{c, w^{2}} \leq C c_{0}^{2}, \\
& w_{k}(x) \geq 3 w\left(I\left(x, 2^{-k}\right)\right) / 4,
\end{aligned}
$$

where $k \geq M$ and

$$
w_{k}(x)=\sum_{I: 2^{-M}>l(I) \geq 2^{-k}} \lambda_{w, I} b_{I}(x)+w_{M}(x) .
$$

Proof. Take the same $\varphi(x)$ as in the proof of Lemma 8.1. If $l(I)<$ $2^{-M}$, then set

$$
\lambda_{w, I}=|I|^{-1 / 2}\left(\iint_{T(I)}\left|\varphi_{t} * w(y)\right|^{2} t^{-1} d t d y\right)^{1 / 2}
$$

and

$$
b_{I}(x)=\iint_{T(I)} \varphi_{t}(x-y)\left(\varphi_{t} * w\right)(y) t^{-1} d t d y / \lambda_{w, I}
$$


If $l(I) \geq 2^{-M}$, then set $\lambda_{w, I}=0$ and $b_{I}(x)=0$. Set

$$
w_{M}(x)=w(x)-\sum_{I: l(I)<2^{-M}} \lambda_{w, I} b_{I}(x) .
$$

Then (8.11)-(8.12) are clear. Conditions (8.13)-(8.16) follow from Lemma 4.2 and the same argument as in the proof of Lemma 8.1.

Let $k \geq M$. Take the same $\Phi$ as in the proof of Lemma 8.2. Then

$$
w_{k}=w * 2^{k n} \Phi\left(2^{k}\right) \text {. }
$$

Put $J=I\left(x, 2^{-k}\right)$. Since

$$
\begin{aligned}
\left|w(J)-w_{k}(x)\right| & =\left|\int(w(J)-w(y)) 2^{k n} \Phi\left(2^{k}(x-y)\right) d y\right| \\
& \leq C \int_{2 J}|w(J)-w(y)| d y /|J| \leq C c_{0} w(J)
\end{aligned}
$$

by (4.2)-(4.3), we get (8.17).

LEMma 8.5. Let $j$ be a positive integer. Assume that $\left\{\mathbf{b}_{I}(x)\right\}_{I: \text { dyadic }}$ and $\left\{\lambda_{I}\right\}_{I: \text { dyadic }}$ satisfy (8.4), (8.6),

$$
\operatorname{supp} \mathbf{b}_{I} \subset 2^{j} I
$$

and

$$
\left\|\mathbf{b}_{I}\right\|_{\text {Lip } 1} \leq\left(2^{j} l(I)\right)^{-1}
$$

Let $\alpha>0$. Set

$$
\mathbf{f}(x)=\sum_{I: I(I)<\alpha} \lambda_{I} \mathbf{b}_{I}(x)
$$

Then

(8.20)

$$
\|\mathbf{f}\|_{\mathrm{BMO}_{w}} \leq C 2^{j n}
$$

where $C$ is independent of $\alpha$.

Proof. Take any cube $J$ (not necessarily dyadic). Let $2^{-k} \leq l(J)<$ $2^{-k+1}$. Set

$$
\tilde{\mathbf{f}}=\sum_{I: l(I) \geq 2^{-J-k+1}} \lambda_{I} \mathbf{b}_{I}
$$

and

$$
\tilde{\mathbf{f}}=\sum_{\substack{I: l(I)<2^{-J-k+1}, I \cap 3 J \neq \varnothing}} \lambda_{I} \mathbf{b}_{I}
$$


Then

$$
\mathbf{f}=\tilde{\mathbf{f}}+\tilde{\mathbf{f}} \text { on } J \text {. }
$$

Note that

$$
\begin{aligned}
|\tilde{\mathbf{f}}(x)-\tilde{\mathbf{f}}(y)| & \leq \sum_{h=-\infty}^{j+k-1} \sum_{l(I)=2^{-h}} \lambda_{I}\left|\mathbf{b}_{I}(x)-\mathbf{b}_{I}(y)\right| \\
& \leq \sum \sum \lambda_{I} 2^{-j+h}|x-y| \chi_{2^{j+1} J}(x) \\
& \leq 2^{k}|x-y| \sum \sum \lambda_{I} 2^{-j-k+h} \chi_{2^{j+1} I}(x) \\
& \leq 2^{k}|x-y| 2^{j n} w\left(I\left(x, 2^{-k}\right)\right)
\end{aligned}
$$

provided $|x-y|<2^{-k}$. By (8.4), (8.6), (8.18), (8.19) and by Lemma 3.3 of [32] we get

$$
\begin{aligned}
\|\tilde{\mathbf{f}}\|_{L^{2}} & \leq C 2^{j n}\left(\sum_{\substack{I: l(I)<2^{-j-k+1}, I \cap 3 J \neq \varnothing}} \lambda_{I}^{2}|I|\right)^{1 / 2} \\
& \leq C 2^{j n} w(J)|J|^{1 / 2} .
\end{aligned}
$$

Thus by $(8.21)-(8.23)$

$$
\begin{aligned}
& \int_{J}\left|\mathbf{f}(x)-\tilde{\mathbf{f}}\left(x_{J}\right)\right| d x / m_{w}(J) \\
& \leq C\left(\int_{J}\left|\mathbf{f}(x)-\tilde{\mathbf{f}}\left(x_{J}\right)\right|^{2} d x /|J|\right)^{1 / 2} / w(J) \leq C 2^{\prime n} .
\end{aligned}
$$

9. Proof of the Main Lemma in $§ 4$. We may assume $t=1$ in (4.6) and

$$
w(I(0,1))=1 .
$$

In this section $C_{9.1}$ is a large constant depending only on $\theta_{1}, \ldots, \theta_{m}$. Let $M$ be a large integer depending only on $\theta_{1}, \ldots, \theta_{m}$ and $C_{9.1}$. Let $c_{0}>0$ be small enough depending only on $\theta_{1}, \ldots, \theta_{m}, C_{9.1}$ and $M$. In particular

$$
C_{9.1} 2^{-M}<1 \text { and } C_{9.1}^{4} 2^{M(n+2)} c_{0}<1 .
$$

First, we give a rough explanation of the procedure to construct $\mathbf{g}(x)$. We construct a sequence $\left\{\mathbf{g}_{k}\right\}_{k=M}^{\infty}$ such that

(i) $\left|\mathbf{g}_{k}(x)\right| \leq w_{k}(x) \chi_{I(0,4)}(x)$,

(ii) $\mathbf{f}_{k}-\mathbf{g}_{k}+$ (small errors) $\in S$. 
[For the definitions of $w_{k}$ and $\mathbf{f}_{k}$, recall Lemmas 8.2 and 8.4.] Then by letting $k \rightarrow+\infty$, we get $\tilde{\mathbf{g}}$ such that

$$
\begin{gathered}
|\tilde{\mathbf{g}}(x)| \leq w(x) \chi_{I(0,4)}(x), \\
\mathbf{f}-\tilde{\mathbf{g}}+(\text { small errors }) \in S .
\end{gathered}
$$

Next we estimate the weighted BMO norms of the error terms and repeat the same procedure for them.

In order to meet the condition (i), we must adjust the length of the vector-valued function $\mathbf{g}_{k}$. We must do this adjustment under the restriction (ii). Here we use the property of the space $S$ that was proved in Lemma 6.1.

Now we go into details.

By Lemmas 8.3-8.4, we get

$$
\begin{aligned}
& \mathbf{f}_{M}(x),\left\{\mathbf{b}_{I}(x)\right\}_{I: \text { dyadic }} \text { and }\left\{\lambda_{f, I}\right\}_{I: \text { dyadic }} \\
& w_{M}(x),\left\{b_{I}(x)\right\}_{I: \text { dyadic }} \text { and }\left\{\lambda_{w, I}\right\}_{I: \text { dyadic }}
\end{aligned}
$$

such that (8.9)-(8.10), (8.3)-(8.5), (8.6)'-(8.8)' and (8.11)-(8.17) hold.

Set

$$
\begin{array}{lll}
\lambda_{I}=\lambda_{f, I}+\lambda_{w, I} & \text { if } & l(I)<2^{-M}, \\
\lambda_{I}=c_{0} w(I) & \text { if } & l(I)=2^{-M}, \\
\lambda_{I}=0 & \text { if } & l(I)>2^{-M} .
\end{array}
$$

LEMMA 9.1.

$$
\left\|\sum_{I} \lambda_{I}^{2}|I| \delta_{\left(x_{I}, l(I)\right)}\right\|_{c, w^{2}} \leq C c_{0}^{2}
$$

This is clear from (8.6)' and (8.16).

From these $\left\{\lambda_{I}\right\}_{I}$, we define $\eta_{k}(x)$ and $\varepsilon_{k}(x)$ by Definition 7.2. Then by Lemmas 9.1 and 7.3 we get

LEMMA 9.2. If $x \in I$ and $l(I)=2^{-k}$, then

$$
C \lambda_{I} \leq \eta_{k}(x) \leq \varepsilon_{k}(x) \leq C^{\prime} c_{0} w(I) .
$$

LEMMA 9.3.

$$
\left\|\sum_{k=-\infty}^{\infty} \varepsilon_{k}(x)^{2} \delta_{t=2^{-k}}\right\|_{c, w^{2}} \leq C c_{0}^{2} .
$$


We inductively construct

$$
\left\{\boldsymbol{\varphi}_{k}(x)\right\}_{k=M+1}^{\infty} \text { and } \quad\left\{\boldsymbol{\beta}_{I, j}(x)\right\}_{I: \text { dyadic }, l(I)<2^{-M} ; j=4,5,6, \ldots}
$$

with the following properties (C.1)-(C.8). Put

$$
\begin{aligned}
& \text { (9.6) } \mathbf{p}_{I, 1}(x)=\sum_{j=4}^{M-1} 2^{-j(n+1)} \boldsymbol{\beta}_{I, j}(x), \\
& \text { (9.7) } \mathbf{p}_{I, 2}(x)=\sum_{j=M}^{\infty} 2^{-J(n+1)} \boldsymbol{\beta}_{I, j}(x), \\
& \text { (9.8) } \mathbf{g}_{M}(x)=\mathbf{f}_{M}(x), \\
& \text { (9.9) } \mathbf{g}_{k}(x)=\mathbf{f}_{k}(x)+\sum_{h=M+1}^{k} \sum_{I: l(I)=2^{-h}} \lambda_{I} \mathbf{p}_{I, 1}(x)-\sum_{h=M+1}^{k} \boldsymbol{\varphi}_{h}(x) \\
& \text { for } k=M+1, M+2, \ldots
\end{aligned}
$$

(C.1) $\operatorname{supp} \boldsymbol{\beta}_{I, j} \subset 2^{j} I, \quad\left\|\boldsymbol{\beta}_{I, J}\right\|_{\text {Lip } 1} \leq C_{9.1} 2^{-j} l(I)^{-1}, \int \boldsymbol{\beta}_{I, j} d x=0$,

(C.2) $\quad \boldsymbol{\beta}_{I, j}(x) \equiv 0 \quad$ if $\quad I \cap I(0,4)=\varnothing$,

(C.3) $\mathbf{p}_{I, 1}+\mathbf{p}_{I, 2} \in S$,

(C.4) $\left|\varphi_{k}(x)\right| \leq C_{9.1}^{2} \varepsilon_{k}(x)^{2} 2^{M(n+2)} / w\left(I\left(x, 2^{-k}\right)\right)$,

(C.5) $\left|\boldsymbol{\varphi}_{k}(x)-\boldsymbol{\varphi}_{k}(y)\right| \leq C_{9.1}^{4} c_{0} 2^{M(n+2)} \varepsilon_{k}(x) 2^{k}|x-y|$

provided $|x-y|<2^{-k}$,

(C.6) $\operatorname{supp} \boldsymbol{\varphi}_{k} \subset \operatorname{supp} \mathbf{g}_{k} \subset I\left(0,3+2^{-1}+2^{-2}+\cdots+2^{-k+M}\right)$,

(C.7) $\left|\mathbf{g}_{k}(x)\right| \leq w_{k}(x)$,

(C.8) $\quad\left|\mathbf{g}_{k}(x)-\mathbf{g}_{k}(y)\right| \leq C_{9.1} \varepsilon_{k}(x) 2^{k}|x-y|$ provided $|x-y|<2^{-k}$.

The construction of the above functions is explained at the end of this section. We accept this construction temporarily and prove the Main Lemma. By the same argument as [32], we can show that $\tilde{\mathbf{g}}=\lim _{k \rightarrow \infty} \mathbf{g}_{k}$ exists in $L^{2}$. By (C.6)-(C.7), we get

$$
\operatorname{supp} \tilde{\mathbf{g}} \subset I(0,4)
$$

and

$$
|\tilde{\mathbf{g}}(x)| \leq w(x) .
$$

By (9.9)

(9.12) $\quad \tilde{\mathbf{g}}(x)=\mathbf{f}(x)+\sum_{I: l(I)<2^{-M}} \lambda_{I} \mathbf{p}_{I, 1}(x)-\sum_{h=M+1}^{\infty} \boldsymbol{\varphi}_{h}(x)$. 
Set

$$
\begin{aligned}
& \mathbf{p}_{2}(x)=\sum_{I: I(I)<2^{-M}} \lambda_{I} \mathbf{p}_{I, 2}(x), \\
& \boldsymbol{\varphi}(x)=\sum_{h=M+1}^{\infty} \boldsymbol{\varphi}_{h}(x)
\end{aligned}
$$

LEMMA 9.4.

$$
\mathbf{f}-\left(\tilde{\mathbf{g}}+\mathbf{p}_{2}+\boldsymbol{\varphi}\right) \in S
$$

Proof. Since

$$
\tilde{\mathbf{g}}=\mathbf{f}+\sum_{I: l(I)<2^{-M}} \lambda_{I}\left(\mathbf{p}_{I, 1}+\mathbf{p}_{I, 2}\right)-\mathbf{p}_{2}-\boldsymbol{\varphi}
$$

by (9.12), the lemma follows from (C.3).

\section{LEMMA 9.5.}

$$
\operatorname{supp} \varphi \subset I(0,4) \text {, }
$$

$$
\|\boldsymbol{\varphi}\|_{\mathrm{BMO} w} \leq C C_{9.1}^{4} c_{0}^{2} 2^{M(n+2)} \text {. }
$$

Proof. Condition (9.15) is clear from (C.6). Take any I (not necessarily dyadic). Then

$$
\begin{aligned}
& \int_{I_{k: 2^{-k}<l(I)}}\left|\boldsymbol{\varphi}_{k}(x)\right| d x / m_{w}(I) \\
& \quad \leq C_{9.1}^{2} 2^{M(n+2)} \int_{I_{k: 2^{-k}<l(I)}} \varepsilon_{k}(x)^{2} w\left(I\left(x, 2^{-k}\right)\right)^{-1} d x / m_{w}(I) \\
& \quad \leq C_{9.1}^{2} 2^{M(n+2)} \int_{I} \sum \varepsilon_{k}(x)^{2} w\left(I\left(x, 2^{-k}\right)\right)^{-2} d x /|I| \quad \text { by }(\text { C. } 4) \\
& \quad \leq C C_{9.1}^{2} c_{0}^{2} 2^{M(n+2)}
\end{aligned}
$$

by Lemmas 9.3 and 7.1. On the other hand,

$$
\left|\sum_{k: 2^{-k} \geq l(I)}\left(\varphi_{k}(x)-\boldsymbol{\varphi}_{k}(y)\right)\right| / w(I) \leq C C_{9.1}^{4} c_{0}^{2} 2^{M(n+2)}
$$


if $x, y \in I$ by (C.5). Thus

$$
\int_{I}\left|\sum_{k=M+1}^{\infty} \boldsymbol{\varphi}_{k}(x)-\sum_{2^{-k} \geq l(I)} \boldsymbol{\varphi}_{k}\left(x_{I}\right)\right| d x / m_{w}(I) \leq C C_{9.1}^{4} c_{0}^{2} 2^{M(n+2)} .
$$

Set

$$
\begin{aligned}
& \mathbf{f}_{2}=\boldsymbol{\varphi}, \\
& \mathbf{f}_{3}=\sum_{j=M+1}^{\infty} 2^{-j(n+1)} \sum_{I: l(I) \leq 2^{-j}} \lambda_{I} \boldsymbol{\beta}_{I, j}, \\
& \mathbf{f}_{k}=\sum_{\substack{j, I: l(I)=2^{k-j-3} \\
l(I)<2^{-M}}} 2^{-j(n+1)} \lambda_{I} \boldsymbol{\beta}_{I, j}, \quad k \geq 4 .
\end{aligned}
$$

LEMMA 9.6.

$$
\sum_{k=2}^{\infty} \mathbf{f}_{k}=\boldsymbol{\varphi}+\mathbf{p}_{2}
$$

LEMMA 9.7. For $k \geq 3$,

$$
\begin{aligned}
& \operatorname{supp} \mathbf{f}_{k} \subset I\left(0,2^{k}\right), \\
& \int \mathbf{f}_{k} d x=0, \\
& \left\|\mathbf{f}_{k}\right\|_{\mathrm{BMO} w} \leq C C_{9.1} c_{0} 2^{-M} 2^{-k(n+1)} .
\end{aligned}
$$

Proof. We show only (9.19). If $k \geq 4$, then

$$
\begin{aligned}
\left\|\mathbf{f}_{k}\right\|_{\mathrm{BMO} w} & \leq \sum_{j=M+k-2}^{\infty} \sum_{I: l(I)=2^{k-j-3}} 2^{-j(n+1)} \lambda_{I}\left\|\boldsymbol{\beta}_{I, j}\right\|_{\mathrm{BMO} w} \\
& \leq \sum_{j} \sum_{I} 2^{-j(n+1)} C c_{0} \leq \sum_{j} 2^{-j(n+1)} C c_{0} 2^{(j-k) n} \\
& \leq C c_{0} 2^{-M} 2^{-k(n+1)} .
\end{aligned}
$$

If $k=3$, then

$$
\begin{aligned}
\left\|\mathbf{f}_{3}\right\|_{\mathrm{BMO} w} & =\sum_{j=M+1}^{\infty} 2^{-j(n+1)}\left\|_{I: l(I) \leq 2^{-j}} \lambda_{I} \boldsymbol{\beta}_{I, j}\right\|_{\mathrm{BMO} w} \\
& \leq \sum_{j=M+1}^{\infty} 2^{-j} C c_{0} \leq C c_{0} 2^{-M} \text { by Lemmas 8.5 and 9.1. }
\end{aligned}
$$


From Lemmas 9.4-9.7, we obtain the following.

Lemma 9.8. Assume the hypothesis of the Main Lemma. Then there exist $\tilde{\mathbf{g}}(x)$ and $\left\{\mathbf{f}_{j}(x)\right\}_{j=2}^{\infty}$ such that

$$
\mathbf{f}-\left(\tilde{\mathbf{g}}+\sum_{j=2}^{\infty} \mathbf{f}_{j}\right) \in S,
$$$$
\operatorname{supp} \mathbf{f}_{j} \subset I\left(0,2^{j} t\right) \text {, }
$$$$
\left\|\mathbf{f}_{j}\right\|_{\mathrm{BMO} w} \leq c_{0} \alpha\left(M, c_{0}\right) 2^{-j(n+1)} \text {, }
$$$$
|\tilde{\mathbf{g}}(x)| \leq w(x) \text {, }
$$$$
\operatorname{supp} \tilde{\mathbf{g}} \subset I(0,4 t) \text {, }
$$

where

$$
\alpha\left(M, c_{0}\right)=C\left(C_{9.1} 2^{-M}+C_{9.1}^{4} c_{0} 2^{M(n+2)}\right) .
$$

Since we have assumed $t=1$ at the beginning of this section, we showed the above only for the case $t=1$. But the general case follows easily from the case $t=1$.

Proof of the Main Lemma. We continue to assume $t=1$. Take $M$ and $c_{0}$ so that

$$
1+\alpha\left(M, c_{0}\right)<2^{1 / 4} .
$$

Applying Lemma 9.8 to $\mathbf{f}$, we obtain $\tilde{\mathbf{g}}$ and $\left\{\mathbf{f}_{j}\right\}_{j=2}^{\infty}$ with (9.20)-(9.24). Next, applying Lemma 9.8 to each $\mathbf{f}_{j}$, we obtain $\tilde{\mathbf{g}}_{j}$ and $\left\{\mathbf{f}_{j, k}\right\}_{k=2}^{\infty}$. Repeating this process, we obtain $\left\{\tilde{\mathbf{g}}_{j_{1}, \ldots, j_{i}}\right\}$ and $\left\{\mathbf{f}_{j_{1}, \ldots, j_{l}}\right\}$ such that

$$
\begin{aligned}
& \mathbf{f}_{j_{1}, \ldots j_{i}}-\left(\tilde{\mathbf{g}}_{j_{1}, \ldots j_{l}}+\sum_{k=2}^{\infty} \mathbf{f}_{j_{1}, \ldots j_{l}, k}\right) \in S, \\
& \operatorname{supp} \mathbf{f}_{j_{1}, \ldots j_{l}, k} \subset I\left(0,2^{j_{1}+\cdots+j_{l}+k}\right), \\
& \left\|\mathbf{f}_{j_{1}, \ldots j_{l}, k}\right\|_{\mathrm{BMO} w} \leq c_{0} \alpha^{i+1} 2^{-\left(j_{1}+\cdots+j_{l}+k\right)(n+1)}, \\
& \left|\tilde{\mathbf{g}}_{j_{1}, \ldots j_{l}}(x)\right| \leq \alpha^{i} 2^{-\left(j_{1}+\cdots+j_{l}\right)(n+1)} w(x), \\
& \operatorname{supp} \tilde{\mathbf{g}}_{j_{1}, \ldots j_{l}} \subset I\left(0,4 \cdot 2^{j_{1}+\cdots+j_{l}}\right) .
\end{aligned}
$$

Set

$$
\mathbf{g}^{i}=\tilde{\mathbf{g}}+\sum_{s=1}^{i} \sum_{j_{1}, \ldots, j_{s}} \tilde{\mathbf{g}}_{j_{1}, \ldots, j_{s}} .
$$


Then

$$
\mathbf{f}-\left(\mathbf{g}^{i}+\sum_{j_{1}, \ldots, j_{l+1}} \mathbf{f}_{j_{1}, \ldots, j_{l}+1}\right) \in S .
$$

Set

$$
\mathbf{g}=\lim _{i \rightarrow \infty} \mathbf{g}^{i}
$$

Since $\sum_{j_{1}, \ldots, j_{i+1}} \mathbf{f}_{j_{1}, \ldots, j_{i+1}}$ tends to 0 in $L^{2}$ as $i \rightarrow \infty$, $\mathbf{g}$ satisfies (4.8). On the other hand,

$$
\begin{aligned}
\mathbf{g} & =\tilde{\mathbf{g}}+\sum_{k=1}^{\infty} \sum_{s: 1 \leq s \leq k / 2} \sum_{j_{1}, \ldots, j_{s}: j_{1}+\cdots+j_{s}=k} \tilde{\mathbf{g}}_{j_{1}, \ldots, j_{s}} \\
& =\tilde{\mathbf{g}}+\sum_{k=1}^{\infty}(9.26)_{k}
\end{aligned}
$$

and

$$
\begin{gathered}
\operatorname{supp}(9.26)_{k} \subset I\left(0,4 \cdot 2^{k}\right), \\
\left|(9.26)_{k}\right| \leq 2^{-k(n+1)} w(x) \sum_{s: 1 \leq s \leq k / 2} \alpha^{s}\left(\begin{array}{c}
k+s-1 \\
s-1
\end{array}\right) \leq 2^{-k(n+1 / 2)} w(x)
\end{gathered}
$$

by (9.25). Thus, (4.7) holds.

Construction of $\left\{\boldsymbol{\beta}_{I, j}\right\}$ and $\left\{\boldsymbol{\varphi}_{k}\right\}$.

We construct these functions inductively. We define $\mathbf{g}_{M}$ by (9.8). Then

$$
\operatorname{supp} \mathbf{g}_{M} \subset I(0,3)
$$

by $(8.7)^{\prime}$. Since

$$
w_{M}(x) \geq C 2^{-C c_{0} M} w(I(0,1))
$$

by (4.3) and (8.17) and since

$$
\left|\mathbf{f}_{M}(x)\right| \leq C c_{0} M,
$$

we get

(9.28)

$$
\left|\mathbf{g}_{M}(x)\right| \leq w_{M}(x)
$$

if $c_{0}>0$ is small enough depending on $M$. By (8.8) and (9.4)

$$
\left|\mathbf{g}_{M}(x)-\mathbf{g}_{M}(y)\right| \leq C \varepsilon_{M}(x) 2^{M}|x-y|
$$

provided $|x-y|<2^{-M}$. [Recall that $\varepsilon_{k}(x)$ is defined by Definition 7.2 from $\left\{\lambda_{I}\right\}$ defined by (9.3)-(9.5).] 
Let $k>M$. Suppose that

$$
\left\{\boldsymbol{\beta}_{I, j}\right\}_{2^{-M}>I(I)>2^{-k}, j=4,5,6, \ldots} \text { and }\left\{\boldsymbol{\varphi}_{h}\right\}_{h=M+1, \ldots, k-1}
$$

have been constructed and that $\mathbf{g}_{k-1}$ defined by (9.8)-(9.9) satisfies

(C.6) $\quad \operatorname{supp}_{k-1} \subset I\left(0,3+2^{-1}+2^{-2}+\cdots+2^{-(k-1-M)}\right)$,

(C.7)' $\left|\mathbf{g}_{k-1}(x)\right| \leq w_{k-1}(x)$,

$(\mathrm{C} .8)^{\prime} \quad\left|\mathbf{g}_{k-1}(x)-\mathbf{g}_{k-1}(y)\right| \leq C_{9.1} \varepsilon_{k-1}(x) 2^{k-1}|x-y|$

$$
\text { provided }|x-y|<2^{-k+1} \text {. }
$$

Notice that by (9.27)-(9.29) $\mathbf{g}_{M}$ satisfies the above (C.6)-(C.8).

LEMMA 9.9. If $|x-y| \leq 2^{M-k}$, then

$$
\left|\mathbf{g}_{k-1}(x)-\mathbf{g}_{k-1}(y)\right| \leq C_{9.1} 2^{M(n+1)} \varepsilon_{k-1}(x) 2^{k}|x-y| .
$$

This follows from (C.8)' and (7.3).

Set

$$
\tilde{w}_{k}(x)=\left|\mathbf{g}_{k-1}(x)\right|+\sum_{I:(9.31)} \lambda_{w, I} b_{I}(x)
$$

where $\Sigma$ is taken over all dyadic cubes $I$ such that

$$
\begin{aligned}
& l(I)=2^{-k} \text { and } \\
& I \cap I\left(0,3+2^{-1}+2^{-2}+\cdots+2^{-(k-1-M)}\right) \neq \varnothing .
\end{aligned}
$$

[Recall that $\left\{b_{I}\right\}$ and $\left\{\mathbf{b}_{I}\right\}$ are defined by Lemmas 8.3-8.4.]

LEMMA 9.10. If $|x-y|<2^{-k}$, then

$$
\left|\tilde{w}_{k}(x)-\tilde{w}_{k}(y)\right| \leq C C_{9.1} \varepsilon_{k}(x) 2^{k}|x-y| .
$$

This follows from (C.8)', the first two inequalities in Lemma 9.2 and (8.13)-(8.15).

From now we explain how to construct

$$
\left\{\boldsymbol{\beta}_{I, j}\right\}_{l(I)=2^{-k}, j=4,5,6, \ldots} \text { and } \boldsymbol{\varphi}_{k} \text {. }
$$

For each $I$ with (9.31) we apply Lemma 6.1 to

$$
\begin{gathered}
\nu=U\left(\mathbf{g}_{k-1}\left(x_{I}\right)\right), \\
b(x)=\lambda_{w, I}^{\prime} b_{I}(x)-\left\langle\lambda_{f, I}^{\prime} \mathbf{b}_{I}(x), \nu\right\rangle,
\end{gathered}
$$


where

$$
\begin{aligned}
\lambda_{w, I}^{\prime} & =\lambda_{w, I} / \lambda_{I}, \\
\lambda_{f, I}^{\prime} & =\lambda_{f, I} / \lambda_{I} .
\end{aligned}
$$

[For the sake of convenience, we define $U(\mathbf{0})=(1,0, \ldots, 0)$ and $0 / 0=0$.] Then we get $\mathbf{p}_{I}(x)$ satisfying (6.4)-(6.5), (6.7)-(6.8) and

$(6.6)^{\prime}\left\langle\mathbf{p}_{I}(x), U\left(\mathbf{g}_{k-1}\left(x_{I}\right)\right)\right\rangle=\lambda_{w, I}^{\prime} b_{I}(x)-\left\langle\lambda_{f, I}^{\prime} \mathbf{b}_{I}(x), U\left(\mathbf{g}_{k-1}\left(x_{I}\right)\right)\right\rangle$.

Applying Lemma 6.2 to $\mathbf{p}_{I}(x)$, we get $\left\{\boldsymbol{\beta}_{I, j}\right\}_{j=4}^{\infty}$. Define $\mathbf{p}_{I, 1}(x)$ and $\mathbf{p}_{I, 2}(x)$ by (9.6)-(9.7). Then (C.1)-(C.3) are clear.

Set

$$
\begin{aligned}
& \mathbf{q}_{I}(x)=\mathbf{p}_{I, 1}(x)+\lambda_{f, I}^{\prime} \mathbf{b}_{I}(x), \\
& \mathbf{h}(x)=\sum_{I:(9.31)} \lambda_{I} \mathbf{q}_{I}(x), \\
& \mathbf{k}(x)=\mathbf{g}_{k-1}(x)+\mathbf{h}(x) .
\end{aligned}
$$

Then

(9.35) $\quad \operatorname{supp} \mathbf{q}_{I} \subset 2^{M-1} I$,

(9.36) $\quad\left|\mathbf{q}_{I}(x)\right| \leq C\left(1+2^{k}\left|x-x_{I}\right|\right)^{-n-1}$,

(9.37) $\quad\left|\mathbf{q}_{I}(x)-\mathbf{q}_{I}(y)\right| \leq C 2^{k}|x-y|\left(1+2^{k}\left|x-x_{I}\right|\right)^{-n-2}$

$$
\text { provided that }|x-y|<2^{-k} \text {, }
$$

(9.38) $|\mathbf{h}(x)| \leq \sum_{I:(9.31)} \lambda_{I}\left|\mathbf{q}_{I}(x)\right| \leq C \eta_{k}(x) \quad$ by (9.36),

(9.39) $\quad|\mathbf{h}(x)-\mathbf{h}(y)| \leq \sum \lambda_{I}\left|\mathbf{q}_{I}(x)-\mathbf{q}_{I}(y)\right| \leq C \eta_{k}(x) 2^{k}|x-y|$

$$
\text { provided }|x-y|<2^{-k} \text { by }(9.37) \text {, }
$$

$$
\operatorname{supp} \mathbf{k} \subset I\left(0,3+2^{-1}+2^{-2}+\cdots+2^{-k+M}\right)
$$

$$
\text { by }(\text { C.6)' and (9.35), }
$$

$$
\begin{aligned}
|\mathbf{k}(x)-\mathbf{k}(y)| & \leq\left|\mathbf{g}_{k-1}(x)-\mathbf{g}_{k-1}(y)\right|+|\mathbf{h}(x)-\mathbf{h}(y)| \\
& \leq\left(C_{9.1} \varepsilon_{k-1}(x) / 2+C \eta_{k}(x)\right) 2^{k}|x-y| \\
& \leq \frac{3}{4} C_{9.1} \varepsilon_{k}(x) 2^{k}|x-y|
\end{aligned}
$$

$$
\text { provided }|x-y|<2^{-k} \text { by }(\text { C.8) })^{\prime} \text { and (9.39) }
$$

since $C_{9.1}$ is large enough. 
Set

(9.42)

$$
\mathbf{k}_{1}(x)=\left\langle\mathbf{k}(x), U\left(\mathbf{g}_{k-1}(x)\right)\right\rangle U\left(\mathbf{g}_{k-1}(x)\right)
$$

and

$$
\begin{aligned}
\mathbf{k}_{2}(x) & =\mathbf{k}(x)-\mathbf{k}_{1}(x) \\
& =\mathbf{h}(x)-\left\langle\mathbf{h}(x), U\left(\mathbf{g}_{k-1}(x)\right)\right\rangle U\left(\mathbf{g}_{k-1}(x)\right) .
\end{aligned}
$$

Then $\mathbf{k}_{1}$ and $\mathbf{k}_{2}$ are orthogonal. Set

$$
v_{I}(x)=\left\langle\mathbf{q}_{I}(x), U\left(\mathbf{g}_{k-1}(x)\right)-U\left(\mathbf{g}_{k-1}\left(x_{I}\right)\right)\right\rangle .
$$

Then

$$
\begin{aligned}
\left\langle\mathbf{q}_{I}(x), U\left(\mathbf{g}_{k-1}(x)\right)\right\rangle= & \left\langle\mathbf{q}_{I}(x), U\left(\mathbf{g}_{k-1}\left(x_{I}\right)\right)\right\rangle+v_{I}(x) \\
= & \left\langle\mathbf{p}_{I, 1}(x), U\left(\mathbf{g}_{k-1}\left(x_{I}\right)\right)\right\rangle \\
& +\left\langle\lambda_{f, I}^{\prime} \mathbf{b}_{I}(x), U\left(\mathbf{g}_{k-1}\left(x_{I}\right)\right)\right\rangle+v_{I}(x) \\
= & \left\langle\mathbf{p}_{I}(x), U\left(\mathbf{g}_{k-1}\left(x_{I}\right)\right)\right\rangle+\cdots+\cdots \\
= & \lambda_{w, I}^{\prime} b_{I}(x)+v_{I}(x)
\end{aligned}
$$

by $(9.32)$ and $(6.6)^{\prime}$. Thus

$$
\begin{aligned}
& \mathbf{k}_{1}(x)=\mathbf{g}_{k-1}(x)+\left\langle\mathbf{h}(x), U\left(\mathbf{g}_{k-1}(x)\right)\right\rangle U\left(\mathbf{g}_{k-1}(x)\right) \\
&=\tilde{w}_{k}(x) U\left(\mathbf{g}_{k-1}(x)\right)+\sum_{I:(9.31)} \lambda_{I} v_{I}(x) U\left(\mathbf{g}_{k-1}(x)\right) \\
& \text { by }(9.33),(9.45) \text { and }(9.30) .
\end{aligned}
$$

Take any dyadic cube $J$ with $l(J)=2^{-k}$.

LEMMA 9.11. (i) If

$$
\left|\mathbf{g}_{k-1}\left(x_{J}\right)\right| \leq 3 w_{k-1}\left(x_{J}\right) / 4,
$$

then

$$
|\mathbf{k}(x)| \leq 7 w_{k}(x) / 8 \text { on } J
$$

(ii) If

$$
\left|\mathbf{g}_{k-1}\left(x_{J}\right)\right| \geq w_{k-1}\left(x_{J}\right) / 2 \text {, }
$$

then

$$
\left|\mathbf{g}_{k-1}(x)\right| \geq w(J) / 4 \text { on } 2^{M} J
$$


Proof. By (8.17) and Lemma 9.2,

$$
w_{k}(x)>\left(1-C c_{0}\right) w_{k-1}(x) .
$$

By (9.38) and Lemma 9.2,

$$
|\mathbf{h}(x)| \leq C c_{0} w_{k}(x) .
$$

Thus (i) holds since $c_{0}$ is small enough.

Let $x, y \in 2^{M} J$. Then, by Lemmas 9.9 and 9.2

$$
\left|\mathbf{g}_{k-1}(x)-\mathbf{g}_{k-1}(y)\right| \leq C C_{9.1} 2^{M(n+2)} c_{0} w(J) .
$$

Since $c_{0}$ is small enough depending on $M$ and $C_{9.1}$, (ii) follows from (8.17).

LEMMA 9.12. If (9.48) holds and if $\left|x-x_{J}\right|,\left|y-x_{J}\right| \leq 2^{M-k}$, then

$$
\begin{aligned}
& \left|U\left(\mathbf{g}_{k-1}(x)\right)-U\left(\mathbf{g}_{k-1}(y)\right)\right| \\
& \leq C C_{9.1} 2^{M(n+1)} \varepsilon_{k-1}(x) 2^{k-1}|x-y| / w(J),
\end{aligned}
$$

(9.50) $|U(\mathbf{k}(x))-U(\mathbf{k}(y))| \leq C C_{9.1} 2^{M(n+1)} \boldsymbol{\varepsilon}_{k}(x) 2^{k}|x-y| / w(J)$.

The first inequality follows from Lemma 9.9 and part (ii) of Lemma 9.11. The second inequality follows from (9.41) and part (ii) of Lemma 9.11 .

LEMMA 9.13. If (9.48) holds, then

(9.51) $\left|v_{I}(x)\right| \leq C C_{9.1} 2^{M(n+2)} \varepsilon_{k-1}(x)\left(1+2^{k}\left|x-x_{I}\right|\right)^{-n-1} / w(J) \quad$ on $J$,

(9.52) $\left|v_{I}(x)-v_{I}(y)\right|$

$$
\leq C C_{9.1} 2^{M(n+2)} \varepsilon_{k-1}(x) 2^{k}|x-y|\left(1+2^{k}\left|x-x_{I}\right|\right)^{-n-1} / w(J) \text { on } J,
$$

(9.53) $\left|\sum_{I:(9.31)} \lambda_{I} v_{I}(x)\right| \leq C C_{9.1} 2^{M(n+2)} \varepsilon_{k-1}(x) \eta_{k}(x) / w(J) \quad$ on $J$,

(9.54) $\left|\sum_{I:(9.31)} \lambda_{I} v_{I}(x)-\sum_{I:(9.31)} \lambda_{I} v_{I}(y)\right|$

$$
\leq C C_{9.1} 2^{M(n+2)} \varepsilon_{k-1}(x) \eta_{k}(x) 2^{k}|x-y| / w(J) \quad \text { on } J \text {. }
$$

Proof. (9.51) follows from (9.35)-(9.36) and (9.49). Note that

$$
\begin{aligned}
v_{I}(x)-v_{I}(y)= & \left\langle\mathbf{q}_{I}(x)-\mathbf{q}_{I}(y), U\left(\mathbf{g}_{k-1}(x)\right)-U\left(\mathbf{g}_{k-1}\left(x_{I}\right)\right)\right\rangle \\
& +\left\langle\mathbf{q}_{I}(y), U\left(\mathbf{g}_{k-1}(x)\right)-U\left(\mathbf{g}_{k-1}(y)\right)\right\rangle .
\end{aligned}
$$


Condition (9.35), (9.37) and (9.49) take care of the first term and conditions (9.36) and (9.49) take care of the second term. Thus, (9.52) holds. Conditions (9.53)-(9.54) follow from (9.51)-(9.52).

LEMMA 9.14. If (9.48) holds, then

$$
\left|\mathbf{k}_{2}(x)\right| \leq C \eta_{k}(x) \text { on } J \text {, }
$$

$$
\left|\mathbf{k}_{2}(x)-\mathbf{k}_{2}(y)\right| \leq C \eta_{k}(x) 2^{k}|x-y| \quad \text { on } J \text {. }
$$

Proof. (9.55) follows from the last formula of (9.43) and (9.38). Note that

$$
\left|U\left(\mathbf{g}_{k-1}(x)\right)-U\left(\mathbf{g}_{k-1}(y)\right)\right| \leq C 2^{k-1}|x-y|
$$

by (9.49), Lemma 9.2 and (9.2). So, (9.56) follows from the last formula of (9.43), (9.39), (9.38) and (9.57).

LEMMA 9.15. If (9.48) holds, then

$$
\begin{aligned}
& || \mathbf{k}(x)\left|-\tilde{w}_{k}(x)\right| \leq C C_{9.1} 2^{M(n+2)} \varepsilon_{k}(x)^{2} / w(J) \quad \text { on } J, \\
& \left|\left(|\mathbf{k}(x)|-\tilde{w}_{k}(x)\right)-\left(|\mathbf{k}(y)|-\tilde{w}_{k}(y)\right)\right| \\
& \quad \leq C C_{91}^{2} 2^{M(n+2)} \varepsilon_{k}(x)^{2} 2^{k}|x-y| / w(J) \quad \text { on } J .
\end{aligned}
$$

Proof. Set $r_{1}(t)=(1+t)^{1 / 2}-1$. Then

$$
\begin{aligned}
& \text { (9.60) }|\mathbf{k}(x)|-\tilde{w}_{k}(x)=\left(\left|\mathbf{k}_{1}(x)\right|^{2}+\left|\mathbf{k}_{2}(x)\right|^{2}\right)^{1 / 2}-\tilde{w}_{k}(x) \\
& =\left\{\left(\tilde{w}_{k}(x)+\sum_{I:(9.31)} \lambda_{I} v_{I}(x)\right)^{2}+\left|\mathbf{k}_{2}(x)\right|^{2}\right\}^{1 / 2}-\tilde{w}_{k}(x) \text { by (9.46) } \\
& =\tilde{w}_{k}(x) r_{1}\left(2 \sum \lambda_{I} v_{I}(x) / \tilde{w}_{k}(x)\right. \\
& \left.\quad+\left(\sum \lambda_{I} v_{I}(x) / \tilde{w}_{k}(x)\right)^{2}+\left(\left|\mathbf{k}_{2}(x)\right| / \tilde{w}_{k}(x)\right)^{2}\right) \\
& =\tilde{w}_{k}(x) r_{2}(x) .
\end{aligned}
$$

Then by (9.53) and (9.55)

$$
\left|r_{2}(x)\right| \leq C C_{9.1} 2^{M(n+2)} \varepsilon_{k}(x)^{2} / w(J)^{2} .
$$

So, (9.58) holds. 
By (9.60), the left-hand side of (9.59)

$$
\begin{aligned}
& \leq\left|\tilde{w}_{k}(x)-\tilde{w}_{k}(y)\right| r_{2}(x)+\tilde{w}_{k}(y)\left|r_{2}(x)-r_{2}(y)\right| \\
& \leq\left|\tilde{w}_{k}(x)-\tilde{w}_{k}(y)\right| r_{2}(x) \\
& +C \tilde{w}_{k}(y)\left\{|| \sum \lambda_{I} v_{I}(x)-\sum \lambda_{I} v_{I}(y) \mid / \tilde{w}_{k}(x)\right. \\
& +\left|\sum \lambda_{I} v_{I}(y)\right|\left|\tilde{w}_{k}(x)^{-1}-\tilde{w}_{k}(y)^{-1}\right| \\
& +\left.|| \mathbf{k}_{2}(x)\right|^{2}-\left|\mathbf{k}_{2}(y)\right|^{2} \mid / \tilde{w}_{k}(x)^{2} \\
& \left.+\left|\mathbf{k}_{2}(y)\right|^{2}\left|\tilde{w}_{k}(x)^{-2}-\tilde{w}_{k}(y)^{-2}\right|\right\} .
\end{aligned}
$$

Lemma 9.10 and (9.61) take care of the first term. Conditions (9.54), (9.53), Lemma 9.10, (9.56) and (9.55) take care of the second term.

Let $t_{k}(x) \geq 0$ be such that

$$
\begin{aligned}
& t_{k}(x)=0 \quad \text { if }\left|\mathbf{g}_{k-1}(x)\right| \leq w_{k-1}(x) / 2, \\
& t_{k}(x)=1 \quad \text { if }\left|\mathbf{g}_{k-1}(x)\right| \geq 3 w_{k-1}(x) / 4, \\
& \left|t_{k}(x)-t_{k}(y)\right| \leq 2^{k}|x-y|
\end{aligned}
$$

Set

$$
\begin{aligned}
& \boldsymbol{\varphi}_{k}(x)=t_{k}(x)\left(|\mathbf{k}(x)|-\tilde{w}_{k}(x)\right) U(\mathbf{k}(x)), \\
& \mathbf{g}_{k}(x)=\mathbf{k}(x)-\boldsymbol{\varphi}_{k}(x) .
\end{aligned}
$$

By (9.32)-(9.34) this definition of $\mathbf{g}_{k}$ coincides with (9.9).

Condition (C.4) follows from (9.62) and (9.58). Condition (C.5) follows from the inequality

$$
\begin{aligned}
\left|\boldsymbol{\varphi}_{k}(x)-\boldsymbol{\varphi}_{k}(y)\right| \leq & \left|t_{k}(x)-t_{k}(y)\right||| \mathbf{k}(x)\left|-\tilde{w}_{k}(x)\right| \\
& +t_{k}(y)\left|\left(|\mathbf{k}(x)|-\tilde{w}_{k}(x)\right)-\left(|\mathbf{k}(y)|-\tilde{w}_{k}(y)\right)\right| \\
& +\left|\boldsymbol{\varphi}_{k}(y)\right||U(\mathbf{k}(x))-U(\mathbf{k}(y))|,
\end{aligned}
$$

when combined with (9.62), (9.64), (9.58), (9.59), (C.4), (9.50), Lemma 9.2 and $c_{0} 2^{M(n+1)}<1$. Condition (C.6) follows from (C.6)', (9.62) and (9.40). Condition (C.7) is clear from the definition of $\varphi_{k}(x)$, part (i) of Lemma 9.11 and (9.58). Condition (C.8) follows from (9.41) and (C.5) if $c_{0}$ is small enough depending on $M$ and $C_{9.1}$. 
10. Proof of Remark 3. In the proof of Main Lemma, if $\mathbf{f}$ is $\mathbf{R}^{m}$-valued, then $\mathbf{f}_{M}$ and $\left\{\mathbf{b}_{I}\right\}$ are $\mathbf{R}^{m}$-valued. By Remark 2.2 of [32], if $\boldsymbol{\nu} \in \mathbf{R}^{m} \cap \Sigma_{2 m-1}$ and $\theta_{j}(\xi)=\bar{\theta}_{j}(-\xi)$ for $j=1, \ldots, m$, then we can take $\mathbf{p}(x)$ in Lemma 6.1 to be $\mathbf{R}^{m}$-valued. Thus, if $\mathbf{f}$ is $\mathbf{R}^{m}$-valued, then we can take $\tilde{\mathbf{g}}$ and $\left\{\mathbf{f}_{j}\right\}$ in Lemma 9.8 to be $\mathbf{R}^{m}$-valued. Thus we can take $\mathbf{g}$ in Main Lemma to be $\mathbf{R}^{m}$-valued.

\section{REFERENCES}

[1] A. P. Calderón, An atomic decomposition of distributions in parabolic $H^{p}$ spaces, Advances in Math., 25 (1977), 216-225.

[2] A. P. Calderón and A. Zygmund, On higher gradients of harmonic functions, Studia Math., 24 (1964), 211-226.

[3] L. Carleson, Interpolations by bounded analytic functions and the corona problem, Ann. of Math., 76 (1962), 547-559.

[4] _ The Corona Theorem, Proceedings of 15th Scandinavian Congress (Oslo, 1968), Springer-Verlag Lecture Notes in Math., No. 118, 121-132.

[5] __, Two remarks on $H^{1}$ and BMO, Advances in Math., 22 (1976), 269-277.

[6] An explicit unconditional basis in $H^{1}$, Bull. Sci. Math., 104 (1980), 405-416.

[7] S-Y. A. Chang and R. Fefferman, $A$ continuous version of duality of $H^{1}$ and $B M O$ on the bidisc, Ann. of Math., 112 (1980), 179-201.

[8] R. Coifman and B. Dahlberg, Singular integral characterization of nonisotropic $H^{p}$ spaces and the F. and M. Riesz theorem, Proc. Symp. Pure Math., 35 (1979), 231-234.

[9] R. Coifman and R. Rochberg, Another characterization of BMO, Proc. Amer. Math. Soc., 79 (1980), 249-254.

[10] R. Coifman and G. Weiss, On subharmonicity inequalities involving solutions of generalized Cauchy-Riemann equations, Studia Math., 36 (1970), 77-83.

[11] __ Extensions of Hardy spaces and their use in analysis, Bull. Amer. Math. Soc., 83 (1977), 569-646.

[12] O. D. Csereteli, On conjugate functions, Mat. Zametki, 22 (1977), 771-781.

[13] C. Fefferman, Characterizations of bounded mean oscillation, Bull. Amer. Math. Soc., 77 (1971), 587-588.

[14] C. Fefferman and E. M. Stein, $H^{p}$ spaces of several variables, Acta Math., 129 (1972), 137-193.

[15] A. Gandulfo, J. Garcia-Cuerva and M. Taibleson, Conjugate system characterization of $H^{1}$; counter examples for the Euclidean plane and local fields, Bull. Amer. Math. Soc., 82 (1976), 83-85.

[16] J. Garnett, Bounded Analytic Functions, Academic Press, New York, 1981.

[17] J. Garnett and P. Jones, The distance in BMO to $L^{\infty}$, Ann. of Math., 108 (1978), 373-393.

[18] R. F. Gundy, On a theorem of F. and M. Riesz and an equation of A. Wald, Indiana Univ. Math. J., 30 (1981), 589-605.

[19] L. Hörmander, $L^{p}$ estimates for (pluri-) subharmonic functions, Math. Scand., 20 (1967), 65-78.

[20] S. Janson, Characterization of $H^{1}$ by singular integral transforms on martingales and $R^{n}$, Math. Scand., 41 (1977), 140-152.

[21] F. John and L. Nirenberg, On functions of bounded mean oscillation, Comm. Pure Appl. Math., 14 (1961), 415-426. 
[22] P. Jones, Constructions with functions of bounded mean oscillation, $\mathrm{Ph} . \mathrm{D}$. Thesis, University of California, 1978.

[23] Carleson measures and the Fefferman-Stein decomposition of BMO $R$, Ann. of Math., 111 (1980), 197-208.

[24] , Factorization of $A_{p}$ weights, Ann. of Math., 111 (1980), 511-530.

[25] $\longrightarrow L^{\infty}$ estimates for the $\bar{\partial}$ problem in a half-plane, to appear in Acta Math.

[26] B. Muckenhoupt and R. Wheeden, Weighted bounded mean oscillation and the Hilbert transform, Studia Math., 54 (1976), 221-237.

[27] On the dual of weighted $H^{1}$ of the half-space, Studia Math., 63 (1978), 57-79.

[28] J. Peetre, On Littlewood's conjecture and Hardy's inequality, A connection with a problem in interpolation spaces, preprint.

[29] E. M. Stein, Singular Integrals and Differentiability Properties of Functions, Princeton University Press, Princeton, New Jersey, 1970.

[30] E. M. Stein and G. Weiss, On the theory of harmonic functions of several variables I, The theory of $H^{p}$ spaces, Acta Math., 103 (1960), 26-62.

[31] A. Uchiyama, A constructive proof of the Fefferman-Stein decomposition of BMO on simple martingales, to appear in the Proceedings of the conference in honor of Antoni Zygmund, held at the University of Chicago, 1981.

[32] A constructive proof of the Fefferman-Stein decomposition of $B M O\left(\mathbf{R}^{n}\right)$, Acta Math., 148 (1982), 215-241.

[33] N. Th. Varopoulos, BMO functions and the $\bar{\partial}$ equation, Pacific J. Math., 71 (1977), 221-273.

[34] _ A theorem of weak type estimates for Riesz transforms and martingale transforms, Ann. Inst. Fourier, 31 (1981), 257-264.

[35] G. Weiss, Some problems in the theory of Hardy spaces, Proc. Symp. Pure Math., 35 (1979), 189-200.

Received January 11, 1983. Supported in part by NSF MCS-8203319 and Grant-in-Aid for Scientific Research.

TOHOKU UNIVERSITY

Sendai, Miyagi-Ken 980, JaPan 



\title{
PACIFIC JOURNAL OF MATHEMATICS \\ EDITORS
}

\author{
DoNALD BABBITT (Managing Editor) \\ University of California \\ Los Angeles, CA 90024 \\ J. DugunduI \\ University of Southern California \\ Los Angeles, CA 90089-1113 \\ R. FINN \\ Stanford University \\ Stanford, CA 94305 \\ HERMANN FlasChKa \\ University of Arizona \\ Tucson, AZ 85721
}

C. C. Moore

University of California

Berkeley, CA 94720

ARTHUR OGUS

University of California

Berkeley, CA 94720

Hugo Rossi

University of Utah

Salt Lake City, UT 84112

H. SAMELSON

Stanford University

Stanford, CA 94305

ASSOCIATE EDITORS
R. ARENS
E. F. BECKENBACH
B. H. NeUMANN
F. WOLF
K. YoSHIDA (1906-1982)

\section{SUPPORTING INSTITUTIONS}

UNIVERSITY OF ARIZONA
UNIVERSITY OF BRITISH COLUMBIA
CALIFORNIA INSTITUTE OF TECHNOLOGY
UNIVERSITY OF CALIFORNIA
MONTANA STATE UNIVERSITY
UNIVERSITY OF NEVADA, RENO
NEW MEXICO STATE UNIVERSITY
OREGON STATE UNIVERSITY
UNIVERSITY OF ARIZONA
CALIFORNIA INSTITUTE OF TECHNOLOGY
UNIVERSITY OF CALIFORNIA
UNIVERSITY OF NEVADA, RENO
OREGON STATE UNIVERSITY
UNIVERSITY OF OREGON
UNIVERSITY OF SOUTHERN CALIFORNIA
STANFORD UNIVERSITY
UNIVERSITY OF HAWAII
UNIVERSITY OF TOKYO
UNIVERSITY OF UTAH
WASHINGTON STATE UNIVERSITY
UNIVERSITY OF WASHINGTON 


\section{Pacific Journal of Mathematics}

Vol. 115, No. $1 \quad$ September, 1984

Carlos Andradas Heranz and José Manuel Gamboa Mutuberría, A note on projections of real algebraic varieties $\ldots \ldots \ldots \ldots \ldots \ldots \ldots \ldots \ldots \ldots$

Jürgen Appell and Maria Patrizia Pera, Noncompactness principles in nonlinear operator approximation theory $\ldots \ldots \ldots \ldots \ldots \ldots \ldots \ldots \ldots \ldots$

Timothy John Carlson, Extending Lebesgue measure by infinitely many

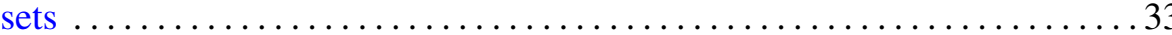

Donald S. Coram and Paul Frazier Duvall, Jr., Non-cell-like

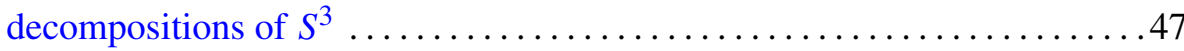

Edward Norman Dancer, Order intervals of selfadjoint linear operators and

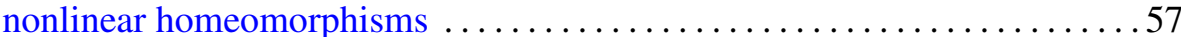

Ralph Jay De Laubenfels, Well-behaved derivations on $C[0,1] \ldots \ldots \ldots 73$

D. Feyel and A. de La Pradelle, Sur certaines extensions du théorème

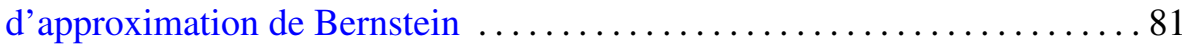

Colin C. Graham and Bertram Manuel Schreiber, Bimeasure algebras on

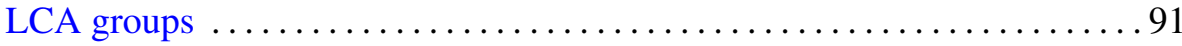

Richard Howard Hudson, Class numbers of imaginary cyclic quartic fields

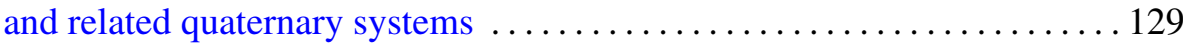

Carl Groos Jockusch, Jr. and Iraj Kalantari, Recursively enumerable sets and van der Waerden's theorem on arithmetic progressions . . . . . . . . 143

J. F. McClendon, On noncontractible valued multifunctions ........... 155 Akihiko Miyachi, Weak factorization of distributions in $H^{p}$ spaces $\ldots \ldots \ldots 165$ Ezzat S. Noussair and Charles Andrew Swanson, Global positive solutions of semilinear elliptic problems

Jon Christopher Snader, Strongly analytic subspaces and strongly

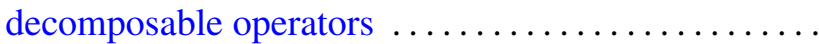

Boguslaw Tomaszewski, A construction of inner maps preserving the Haar measure on spheres

Akihito Uchiyama, The Fefferman-Stein decomposition of smooth functions and its application to $H^{p}\left(\mathbf{R}^{n}\right) \ldots \ldots \ldots$ 